\title{
Deciphering the postsynaptic calcium-mediated energy homeostasis through mitochondria-endoplasmic reticulum contact sites using systems modeling
}

\author{
A. Leung ${ }^{1, *}$, D. Ohadi ${ }^{2, *}$, G. Pekkurnaz ${ }^{3}$, and P. Rangamani ${ }^{2, * *}$ \\ ${ }^{1}$ Chemical Engineering Graduate Program, University of California San Diego, La Jolla, CA, USA \\ ${ }^{2}$ Department of Mechanical and Aerospace Engineering, University of California San Diego, La Jolla, CA, \\ USA \\ ${ }^{3}$ Department of Biological Sciences - Neurobiology, University of California San Diego, La Jolla, CA, USA \\ * Both these authors contributed equally to this manuscript \\ ${ }^{* *}$ Corresponding Author, Department of Mechanical Aerospace Engineering, SME 344G, Jacobs School of \\ Engineering, University of California San Diego, La Jolla, CA 92093-0411 Tel: +1 (858) 534-4734| Email: \\ padmini.rangamani@eng.ucsd.edu
}

\begin{abstract}
Spatiotemporal compartmentation of calcium dynamics is critical for neuronal function, particularly in postsynaptic spines. This exquisite level of $\mathrm{Ca}^{2+}$ compartmentalization is achieved through the storage and release of $\mathrm{Ca}^{2+}$ from various intracellular organelles particularly the endoplasmic reticulum (ER) and the mitochondria. Mitochondria and ER are established storage organelles controlling $\mathrm{Ca}^{2+}$ dynamics in neurons. Mitochondria also generate a majority of energy used within postsynaptic spines to support the downstream events associated with neuronal stimulus. Recently, high resolution microscopy has unveiled direct contact sites between the ER and the mitochondria, which directly channel $\mathrm{Ca}^{2+}$ release from the ER into the mitochondrial membrane. In this study, we develop a computational 3D reaction-diffusion model to investigate the role of MERCs in regulating $\mathrm{Ca}^{2+}$ and ATP dynamics. This spatiotemporal model accounts for $\mathrm{Ca}^{2+}$
\end{abstract}


oscillations initiated by glutamate stimulus of metabotropic and ionotropic glutamate receptors and $\mathrm{Ca}^{2+}$ changes in four different compartments: cytosol, ER, mitochondria, and the MERC microdomain. Our simulations predict that the organization of these organelles and differential distribution of key $\mathrm{Ca}^{2+}$ channels such as $\mathrm{IP}_{3}$ receptor and ryanodine receptor modulate $\mathrm{Ca}^{2+}$ dynamics in response to different stimuli. We further show that the crosstalk between geometry (mitochondria and MERC) and metabolic parameters (cytosolic ATP hydrolysis, ATP generation) influences the cellular energy state. Our findings shed light on the importance of organelle interactions in predicting $\mathrm{Ca}^{2+}$ dynamics in synaptic signaling. Overall, our model predicts that a combination of MERC linkage and mitochondria size is necessary for optimal ATP production in the cytosol.

\footnotetext{
Abbreviations

Endoplasmic reticulum (ER) Inositol 1,4,5-trisphosphate ( $\mathrm{IP}_{3}$ ); Mitochondrial Endoplamic Reticulum Contact (MERC, also MAM) $\mathrm{IP}_{3}$ receptor $\left(\mathrm{IP}_{3} \mathrm{R}\right)$; Ryanodine receptor (RyR); sarcoplasmic/endoplasmic reticulum calcium ATPase (SERCA); Adenine triphosphate (ATP); outer mitochondrial membrane (OMM); intermembrane space (IMS); Mitochondrial Calcium Uniporter (MCU); Plasma Membrane Calcium ATPase (PMCA); inner mitochondrial membrane (IMM); Electron Transport Chain (ETC); Aspartate/glutamate carrier (AGC); Malate/asparate shuttle (MAS); Adenine nucleotide translocator (ANT); Pyruvate dehydrogenase (PDH); Glyceraldehyde 3-phosphate dehydrogenase (GPDH); $\mathrm{Na}^{+} / \mathrm{Ca}^{2+}$ exchanger (NCX); Metabotropic glutamate receptor (mGluR); N-Methyl d-Aspartate Receptor(NMDAR) Protein kinase C (PKC); Diacylglycerol (DAG); Post synaptic density (PSD); Phosphatidylinositol (4,5)-bisphosphate (PIP2).
} 


\section{Introduction}

Compartmentalized calcium handling in postsynaptic structures underlies synaptic communication and controls synaptic plasticity, which is the bidirectional modulation of synaptic strength that is thought to underlie learning and memory formation [1]. In excitatory neurons during synaptic transmission, glutamate released from the presynaptic bouton leads to a localized increase in calcium at the postsynaptic dendrite that is critical for the induction of synaptic plasticity [2,3]. At the postsynaptic site, small bulbous protrusions called dendritic spines act as biochemical computation units that regulate the duration and spread of postsynaptic calcium fluxes produced by glutamatergic neurotransmission [4]. The temporal dynamics of calcium, and the coupling to downstream signaling pathways in dendritic spines depend on many factors, including the nature of stimulus as well as the positioning of the calcium storage organelles, the endoplasmic reticulum (ER) and the mitochondria [5,6]. A continuous tubular network of ER spreads throughout the dendrites and extends into the spines either as a simple smooth tubule or a spine apparatus [7-9]. Mitochondria of various lengths occupy a major portion of dendritic branches and associate closely with the ER particularly at the dendritic base of spines [10-12]. The intimate contact between mitochondria and ER along dendrites allows for a functional inter-organellar coupling and plays a central role in the regulation of the postsynaptic calcium dynamics (Figure 1a). Dysregulation of mitochondria and ER coupling have been demonstrated in neurodegenerative diseases such as Alzheimer's [13-15] and Parkinson's [15, 16], although the underlying mechanisms are yet to be elucidated.

Physical contact sites between mitochondria and ER (Mitochondria-ER Contact Sites, or MERCs) were first observed by electron microscopy, then confirmed by dimerization-dependent fluorescent proteins [17, 18]. MERCs provide a direct avenue for calcium to transfer from the ER to the mitochondria [19]. Because the concentration of ER calcium is several orders of magnitude higher than cytosolic calcium, the existence of these contact sites can result in a direct conduit for increasing mitochondria calcium concentration. These MERCs have been observed to be 2-20\% of a mitochondria's surface area [17]. MERCs are also essential for mitochondrial functions and ATP production [20]. While the exact mechanism for the formation of these sites is unclear, there is evidence that binding proteins link via calcium channels on the surface of the respective organelle $[18,21]$. The role of organelles and MERC in calcium dynamics of dendritic spines is not yet fully understood.

Computational modeling has played a pivotal role in providing insight into calcium dynamics in neurons [22-24]. Bertram et al. [25], Han et al. [26], Wacquier et al. [27] and others have created models addressing the role of calcium in mitochondria. Bertram et al. [25] simplified a mathematical model for 
mitochondrial calcium dynamics in pancreatic beta cells originally developed by Magnus et al. [28] to explain experimentally derived results [29] in which calcium increases NADH under low glucose and reduces NADH in high glucose. Han et al. [26] explicitly modeled the role MERC in a pancreatic beta cell in a compartmental model for the case of a healthy and diabetic cells and predicted a connection between obesity, MERCs, and calcium dynamics. Qi et al. similarly modeled mitochondrial-ER calcium flux into the mitochondria as a function of distance between $\mathrm{IP}_{3} \mathrm{R}$ and $\mathrm{MCU}$ to predict an optimal ER-to-mitochondria distance for the regulation of mitochondrial calcium [30]. However, none of these models are specific to unique circumstances of a dendritic spine or neuronal stimulus patterns. Previously, we and others have shown that spatial modeling of signaling pathways can provide insight into how cell shape and organelle organization can affect the spatiotemporal dynamics of second messengers and signaling cascades [31-36] Although spatial models for neuronal calcium signaling models exist [?,37,38], these do not focus on the metabolic consequences of calcium and incorporate mitochondria.

In this study, we investigated the role of the ER, mitochondria, and MERC in modulating the spatiotemporal dynamics of calcium and localized ATP production in postsynaptic spines using computational modeling. We specifically sought to answer the following questions: What are the spatiotemporal dynamics of $\mathrm{Ca}^{2+}$ and $\mathrm{IP}_{3}$, in response to a glutamate stimulus, in the spine head, spine shaft, and the mitochondria? How does the presence or absence of MERCs affect the dynamics of these second messengers and alter the energy landscape in these locations? And finally, how do different geometric features such as mitochondrial length and MERC surface area ratio affect $\mathrm{Ca}^{2+}$ handling and energy production in spines? To answer these questions, we developed a spatiotemporal model of $\mathrm{Ca}^{2+}$ dynamics in a portion of a dendrite with spatially resolved ER and mitochondria in idealized geometries. We found that the MERC leads to a significant increase in mitochondrial calcium, and subsequently ATP production. Additionally, increasing the surface area of the MERC, increases the calcium influx into the mitochondria, providing insight into how the different extent of MERC can give rise to different calcium states. Finally, we predict metabolic parameters, such as cytosolic hydrolysis, nucleotide transport from the mitochondria to the cytoplasm, and rate of ATP synthesis to be key deciding factors in the delicate balance of calcium signaling and energy homeostasis in the postsynaptic spine.

\section{Results}

We constructed a spatial model with five compartments: the postsynaptic density (PSD), the cytosol, one mitochondria, the ER, and a mitochondria ER Contact region (MERC) (Figure 1a, b). In our simplified 

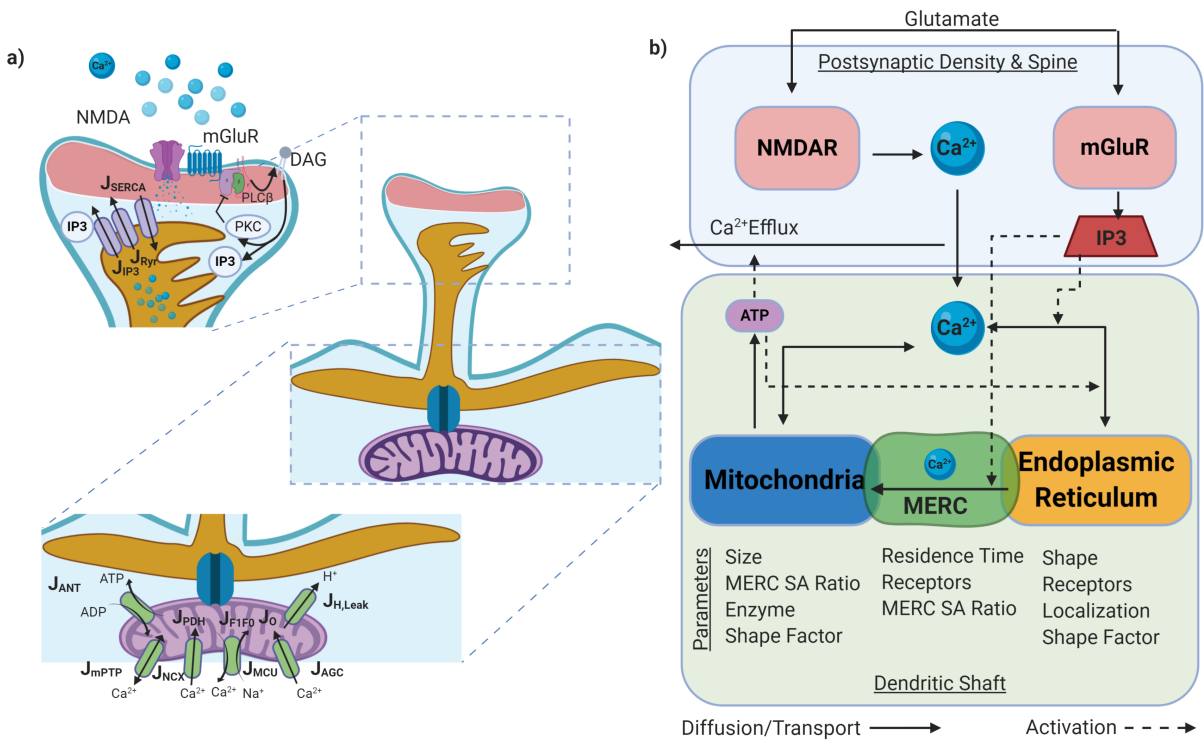

c)

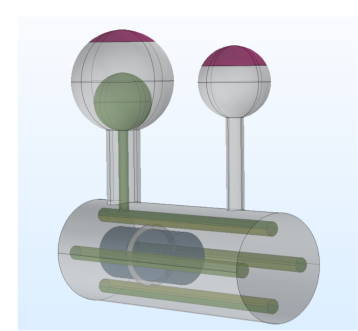

Figure 1: Schematic of the model architecture and fluxes in each compartment. a) Glutamatergic signaling in a postsynaptic spine leads to an influx of $\mathrm{Ca}^{2+}$ from the extracellular space and from the endoplasmic reticulum. Dynamics of $\mathrm{Ca}^{2+}$ have a direct impact on mitochondrial function that alters the ATP production. $[25,28]$. The main fluxes involved in the model are shown here and the mathematical descriptions are given in Table S2. b) The main compartments and investigated parameters in the model are shown in this schematic. In this figure, solid lines represent diffusive or transport fluxes. Dashed lines represent an activation of fluxes. c) Model geometry for a dendritic spine developed using COMSOL Multiphysics ${ }^{\circledR}$. Two spines, one with and one with the spine apparatus are connected by a dendritic shaft containing the ER and mitochondria. PSD, Purple; ER, Green; MERC, Grey; Mitochondria, Blue. SA Ratio refers to the ratio of surface area of MERCs to total mitochondria surface area 
geometry (shown in Figure 1c), the dendritic spine is modeled as a sphere attached to the large cylindrical dendritic shaft by a narrow cylindrical spine neck, geometrically defined in Table S1. Although the morphology of a spine has been shown to govern the magnitude and stability of calcium transients in previous studies [24,31], in this study we simplify complex spine morphology to idealized geometries to focus on the role of mitochondria in neuronal calcium dynamics. While the interconnected ER tublues are widely distributed throughout the cytoplasm, we approximate the ER within the dendritic shaft as long cylinders, keeping ER to cytoplasmic volume ratios constant. The mitochondrion was modeled as a wide cylinder in the center of these four ER. Although dendritic mitochondria have a wide range of sizes from sub micron to to more than $10 \mu \mathrm{m}$ with complex inner membrane and outer membrane morphologies [39], we approximate a relatively small mitochondria of $0.6 \mu \mathrm{m}$ localized at the base of a dendritic spine. The sizes of the compartments in the model are given in Table S1. The details of the model development and numerical methods are given in the supplementary material and in Table S3

\section{Spatial dynamics of $\mathrm{Ca}^{2+}$ and $\mathrm{IP}_{3}$ in spines with and without MERC}

We conducted simulations with a $1 \mathrm{~Hz}$ stimulus of glutamate for $5 \mathrm{~s}$ and ran the simulations until steady state. We also investigated how the two different glutamate receptors, NMDAR and mGluR contributed to the effect of signaling dynamics in the presence and absence of MERC (Figure 2). We dissected these effects by comparing the contribution of NMDAR only, mGluR only, and when both receptors are active. In all simulations, only the larger spine with the spine apparatus was stimulated with the $1 \mathrm{~Hz}$ pulse train. In Figure S1, we instead stimulate the neighboring smaller spine and find minimal differences in dynamics. We conducted these simulations in two geometries - one without the MERCs (no gray ring) and one with MERC consisting of $10 \%$ of the total surface area (Figure 1c). Spatial maps at $1 \mathrm{~s}$ and $5.1 \mathrm{~s}$ were chosen to demonstrate the diffusivity of the $\mathrm{Ca}^{2+}$ and $\mathrm{IP}_{3}$ dynamics (shown in Figure 2). Corresponding temporal dynamics at different locations are shown in Figure S2.

We first looked at $\mathrm{Ca}^{2+}$ dynamics and $\mathrm{IP}_{3}$ distribution with and without MERC (Figure 2). In the presence of MERC, we note that when only NMDAR is active, the calcium dynamics are quite rapid in the stimulated spine and the neighboring spine (Figure 2a). The peak calcium concentration is higher in the spine head $(6 \mu \mathrm{M})$ and about half that in the dendritic shaft $(3 \mu \mathrm{M})$ proximal to the stimulated spine. In contrast, the neighboring spine has a peak $\mathrm{Ca}^{2+}$ concentration of about $4 \mu \mathrm{M}$ with a distinctly lower gradient between spine head and shaft calcium. These results follow from the fact that synaptic activation of NMDAR located at the PSD leads to an increased $\mathrm{Ca}^{2+}$ influx and therefore, the stimulated spine has the peak calcium con- 
centration. When we look at the simulations without MERC (Figure 2b), we note that the absence of MERC has negligible effects on the spine $\mathrm{Ca}^{2+}$ concentration dynamics in both the stimulated and neighboring spines. This is predicted NMDAR affects rapid $\mathrm{Ca}^{2+}$ influx from the spine head and is not immediately affected by organelle calcium dynamics.

When we consider the effect of mGluR response only, we first note that the calcium response is delayed when compared to the stimulus and the NMDAR response in both the stimulated spine and the neighboring spine (Figure 2c). This is because $\mathrm{Ca}^{2+}$ release in this scenario is primarily $\mathrm{IP}_{3}$-mediated and $\mathrm{Ca}^{2+}$ is released from the spine apparatus and the ER. Since the mGluR-only system does not have an influx of calcium, the average concentration of calcium is significantly lower $(1 \mu \mathrm{M})$. Comparing the scenario with and without MERC, we note that the MERC compartment essentially allows for an increased and rapid early release of $\mathrm{Ca}^{2+}$ from the base of the stimulated spine (Figure $2 \mathrm{c}, \mathrm{d}$ ) than in the case without MERC. When we look at the corresponding $\mathrm{IP}_{3}$ dynamics in the stimulated spine (Figure $2 \mathrm{~g}, \mathrm{~h}$ ), we note that the immediate response of the stimulated spine, the spatial gradient of $\mathrm{IP}_{3}$, primarily from the spine head to the dendrite, consistent with the mGluR location and $\mathrm{PIP}_{2}$ hydrolysis from the plasma membrane. The neighboring spine receives $\mathrm{IP}_{3}$ only through diffusion and therefore has a lower concentration and no observable gradients of $\mathrm{IP}_{3}$. When there is no MERC, $\mathrm{IP}_{3}$ concentrations are similar to the case with MERC because $\mathrm{IP}_{3}$ is an early response to mGluR and not affected at these times by $\mathrm{Ca}^{2+}$. In both cases, the neighboring spine shows delayed $\mathrm{IP}_{3}$ dynamics and reduced concentrations.

When both stimuli are included, it becomes immediately obvious that NMDAR contributes dominantly to $\mathrm{Ca}^{2+}$ dynamics (Figure 2e,f) and while $\mathrm{IP}_{3}$ dynamics are dominated by the mGluR activation (Figure 2i,j). We also note that there are spatial differences in the $\mathrm{Ca}^{2+}$ dynamics in the stimulated spine when both NMDAR and mGluR are present as opposed to the individual stimuli because now the $\mathrm{Ca}^{2+}$ influx is both from the PSD and from the spine apparatus.

We also applied no stimulus and found the system quickly approaches an equilibrium for $\mathrm{Ca}^{2+}$, although ATP decay on a longer timescale (Figure S3), discussed in detail below. To further characterize the cytosolic $\mathrm{Ca}^{2+}$ dynamics in response to stimuli in this system, we changed the frequency of the stimulus comparing 1 , 10, and $50 \mathrm{~Hz}$ stimulus over 5 seconds (Figure S4). We found that $\mathrm{Ca}^{2+}$ in the stimulated and neighboring spines showed a frequency dependence for the 1 and $10 \mathrm{~Hz}$ input and an integrated calcium behavior (reading only the lower frequencies for the $50 \mathrm{~Hz}$ ) (Figure S4a, b). 


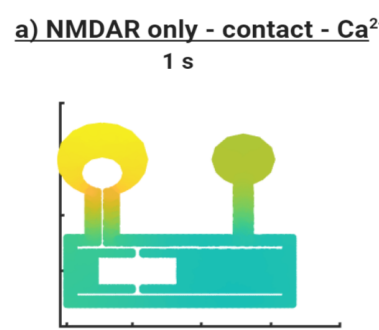

c) mGluR only - contact $-\mathrm{Ca}^{2+}$

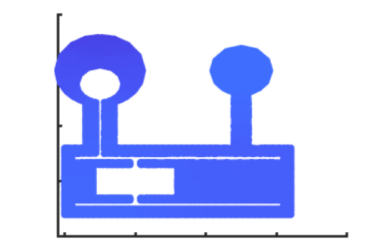

e) Full Model - contact $-\mathrm{Ca}^{2+}$

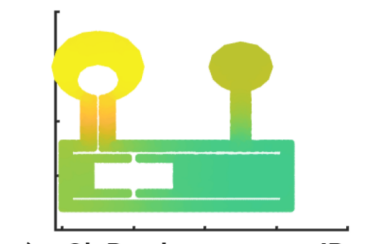

g) mGluR only - contact - $\mathbb{I P}_{3}$

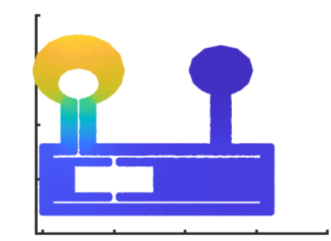

i) Full Model - contact - $\mathbb{P P}_{3}$

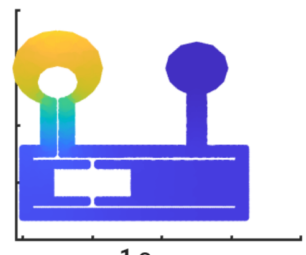

$1 \mathrm{~s}$
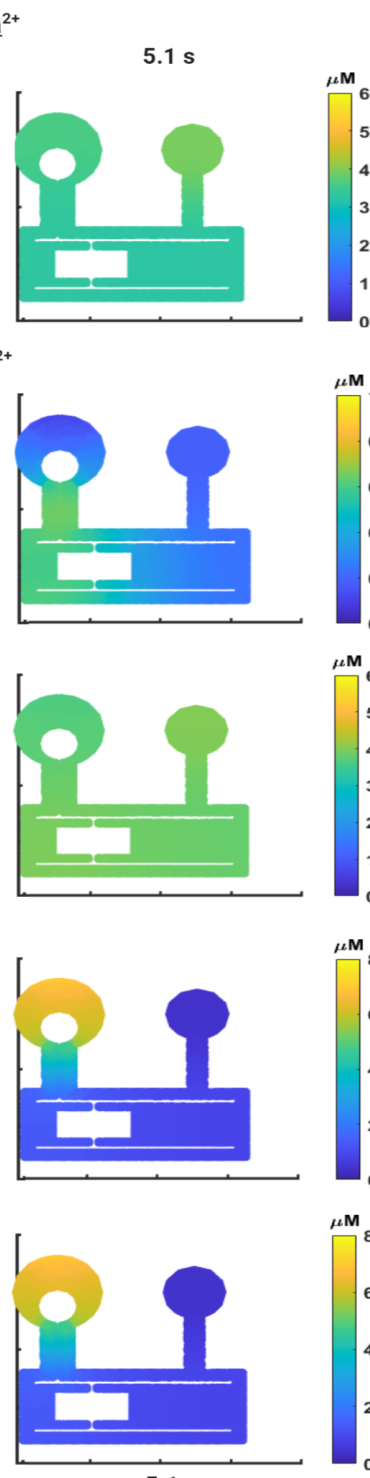

$5.1 \mathrm{~s}$
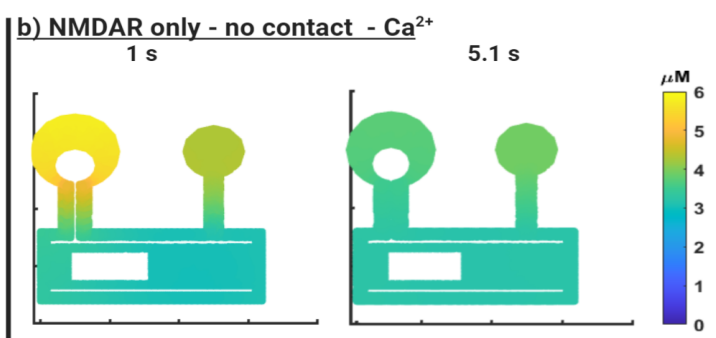

d) mGluR only - no contact $-\mathrm{Ca}^{2+}$
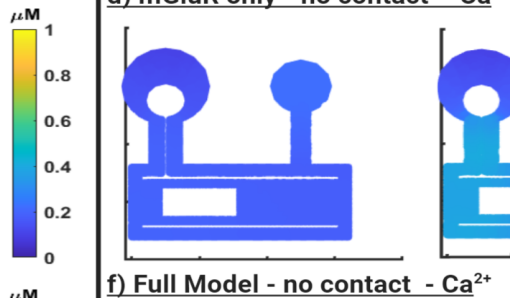

f) Full Model - no contact - $\mathrm{Ca}^{2}$
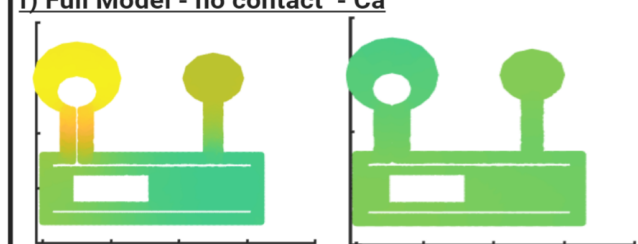

h) mGluR only - no contact - $\mathbb{P}_{3}$
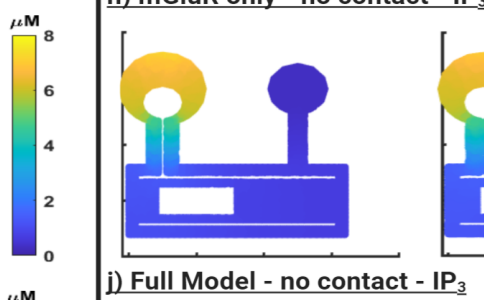

i) Full Model - no contact - IP

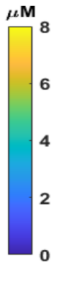

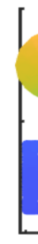
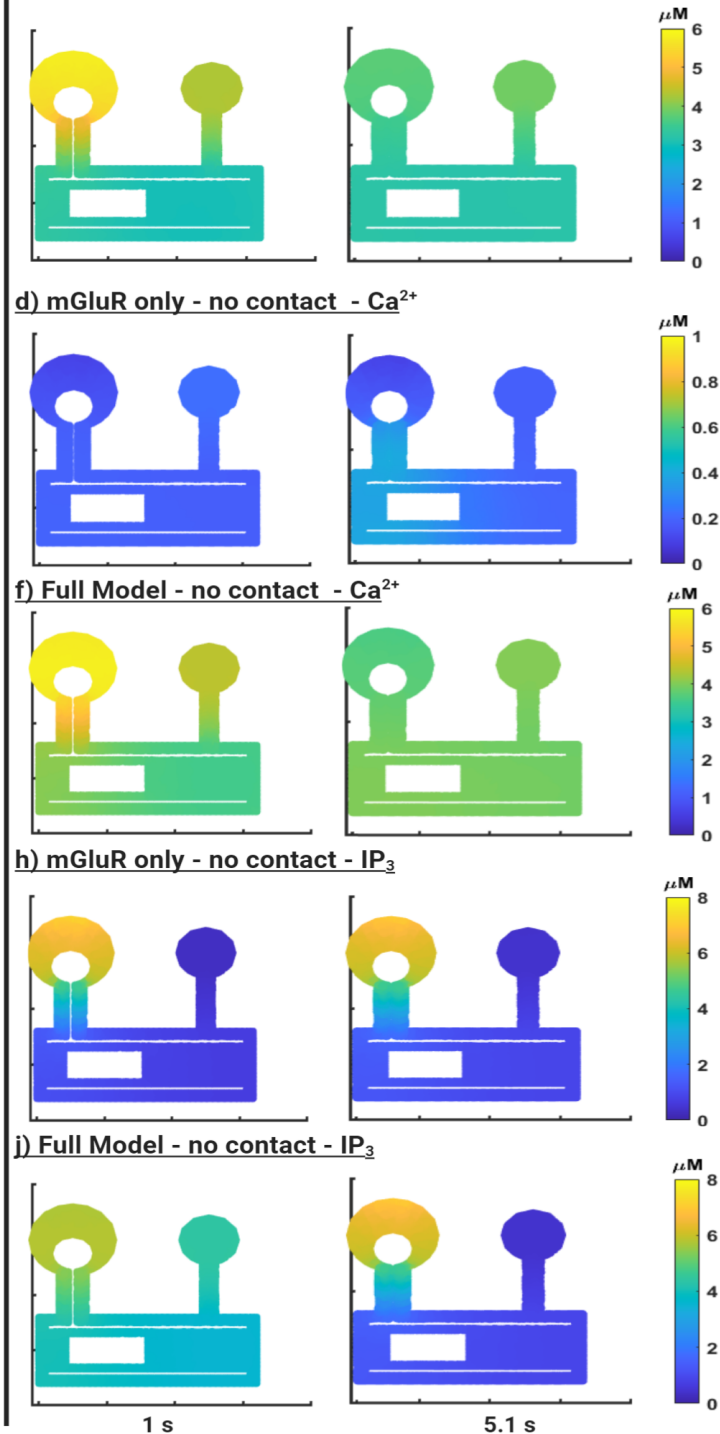

Figure 2: Receptor-based simulations of glutamatergic signaling in spatial calcium model Finite ele-

ment simulations of dendritic spines including mitochondria, ER, and mitochondrial-ER contacts(MERC). a) $\mathrm{Ca}^{2+}$ dynamics in the stimulated and neighboring spine when only NMDAR are stimulated in the presence of MERC. b) Same as (a) in the absence of MERC. In this case, there is no production of $\mathrm{IP}_{3}$. c) $\mathrm{Ca}^{2+}$ dynamics when only mGluR is stimulated in the presence of MERC. d) Same as (c) in the absence of MERC. e) $\mathrm{Ca}^{2+}$ dynamics in the stimulated and neighboring spine when both NMDAR and mGluR are stimulated in the presence of MERC. f) Same as (e) in the absence of MERC. $\mathbf{g}) \mathrm{IP}_{3}$ dynamics in the stimulated and neighboring spines when only mGluR is stimulated in the presence of MERC. h) Same as $(\mathbf{g})$ in the absence of MERC. i) IP 3 dynamics in the stimulated and neighboring spines when both NMDAR and mGluR is stimulated in the presence of MERC. j) Same as (i) in the absence of MERC. 


\section{Presence of MERCs alter organelle $\mathrm{Ca}^{2+}$ dynamics and ATP production}

Next, we investigated the effect of MERCs on $\mathrm{Ca}^{2+}$ dynamics in the ER and mitochondria with the same simulation set as in Figure 2. In the dendrite, the ER acts as an intracellular calcium storage compartment and surface receptors on the ER, such as ryanodine and inositol 1,4,5- trisphosphate $\left(\mathrm{IP}_{3} \mathrm{R}\right)$, rapidly release calcium into the cytosol [40]. Sacroplasmic/endoplasmic reticulum $\mathrm{Ca}^{2+}$-ATPases (SERCAs) can result in high uptake of $\mathrm{Ca}^{2+}$ into the ER (> $300 \mu \mathrm{M}$ [41]) through the consumption of ATP. $\mathrm{Ca}^{2+}$ is then released from the ER through $\mathrm{IP}_{3} \mathrm{R}$ and ryanodine receptors (RyRs) [42]. $\mathrm{Ca}^{2+}$ regulation and mitochondrial ATP production are crucial for synaptic function and neuroplasticity [43]; mitochondria in synaptic terminals aid neurotransmission by producing ATP, buffering calcium, and local protein translation [10,44].

In our simulations, when MERCs are present, we observed that the mitochondrial calcium is significantly higher for all three receptor conditions when compared to the case without MERCs (Figure 3a). This effect was larger when only NMDAR are activated relative to the mGluR or full model. Because MERCs increase the total influx of calcium into the mitochondria, while the efflux is limited by cytosolic exchange, there is a notable accumulation of calcium within the mitochondria. Comparison of mitochondrial calcium dynamics with and without MERCs reveals that the presence of MERCs smooths out the frequency of the glutamate stimulus and time of peak $\mathrm{Ca}^{2+}$ concentration is slightly delayed when compared to the scenario without MERCs. These changes in the calcium dynamics can be better understood by comparing the integrated $\mathrm{Ca}^{2+}$ (area under the curve, AUC), which reveals that the mitochondrial $\mathrm{Ca}^{2+} \mathrm{AUC}$ is higher in the presence of MERCs. This is the first indication from our simulations that spatial compartments such as MERCs can alter $\mathrm{Ca}^{2+}$ dynamics in organelles, consistent with other hypotheses in the literature $[17,45,46]$. On the other hand, no dramatic differences were observed in the $\mathrm{ER} \mathrm{Ca}^{2+}$ dynamics with and without MERC (Figure $3 \mathrm{~b}$ ). Although the MERC is directly connected to the ER and there is significant calcium flux from the ER to the MERC, the relative size of the ER keeps the overall concentration of calcium constant in both cases.

Perhaps the most interesting feature of this model is that MERC act as a $\mathrm{Ca}^{2+}$ microdomain by localizing high $\mathrm{Ca}^{2+}$ concentrations for a finite duration in a receptor dependent manner (Figure 3c). Thus, our model predicts that the presence of MERC plays an important role in mitochondrial and MERC $\mathrm{Ca}^{2+}$ dynamics. As a result, we see an impact on mitochondrial and cytosolic ATP dynamics (Figure 3d,e). In the presence of MERC, both mitochondrial and cytosolic ATP are increased and and have prolonged dynamics. The increased production and prolonged dynamics is dependent on the calcium diffusion within the system. In Figure S5, we show that decreasing the diffusion coefficient leads to a delay in mitochondrial calcium peak, with a corresponding increase in cytosolic and mitochondrial ATP. We also note that compared to the $\mathrm{Ca}^{2+}$ 
dynamics in the ER and the MERC, the mitochondrial $\mathrm{Ca}^{2+}$ and ATP dynamics are smoother, indicating that the mitochondria retain the lower frequency information and not the higher frequency. When we compare the mitochondrial ATP, we note that the presence of MERCs can substantially increase mitochondrial ATP production (Figure 3d) and cytosolic ATP availability (Figure 3e). The dynamics of the mitochondrial membrane potential $\left(\Psi_{m}\right)$ are also altered in the presence of MERCs (Figure 3f), consistent with the mitochondrial ATP dynamics. Interestingly, when we investigated the role of different stimulus frequencies, we found that organelle $\mathrm{Ca}^{2+}$ and ATP dynamics were more sensitive to the change from 1 to $10 \mathrm{~Hz}$ than from the 10 to $50 \mathrm{~Hz}$ (Figure S4c-f). We note that varying the diffusion coefficient of $\mathrm{Ca}^{2+}$ did not alter the results from our model significantly (Figure S5).

Thus, the MERC compartment, which is technically part of the cytosol, is enriched in calcium even though there is no physical membrane separating the two compartments. By serving as a direct conduit for $\mathrm{Ca}^{2+}$ influx from the cytosol, we suggest that MERCs play an important role in localizing $\mathrm{Ca}^{2+}$ and thereby increasing ATP production locally at active synapses.

\section{Effect of mitochondrial size and MERC area fraction on $\mathrm{Ca}^{2+}$ and ATP dynamics}

The mitochondria present in dendrites of neurons are abundant and can vary in size [10,39]. Separately, the area fraction of MERCs are also known to vary [17]. Do these physical variables have an impact on the production of ATP in the mitochondria? To address this question, we conducted two sets of simulations: (i) vary mitochondrial length while maintaining MERC area fraction, and (ii) vary MERC area fraction in mitochondria of 3 sizes (Figures 4 and 5, respectively).

We first explore the effect of mitochondrial size on organelle $\mathrm{Ca}^{2+}$ and ATP dynamics (Figure 4). In these simulations, we maintained the MERC area fraction (calculated as the ratio of MERC surface area in contact with mitochondria to the total mitochondrial surface area) as 0.1 while varying the mitochondrial size from 0.2 to $1.2 \mu \mathrm{m}$. Since the geometries have different volumes, we calculate the AUC as an integral over both time and volume to show the total number of moles of the species integrated over time.

Our simulations showed that increasing mitochondrial size clearly favored increases in mitochondrial $\mathrm{Ca}^{2+}$ (Figure 4a) and MERC $\mathrm{Ca}^{2+}$ (Figure 4b). We note that the increase in the $\mathrm{Ca}^{2+}$ begins to saturate (see peak $\mathrm{Ca}^{2+}$ values in Figure $4 \mathrm{a}$ ) after a certain size of mitochondria suggesting that there is no additional advantage of large mitochondria in terms of $\mathrm{Ca}^{2+}$ concentration. This effect is also seen in the MERC $\mathrm{Ca}^{2+}$. Correspondingly, we observed increases in mitochondrial ATP production capacity but also a saturation at higher mitochondrial sizes (Figure 4c). Thus far, our results are consistent with our expectation that 

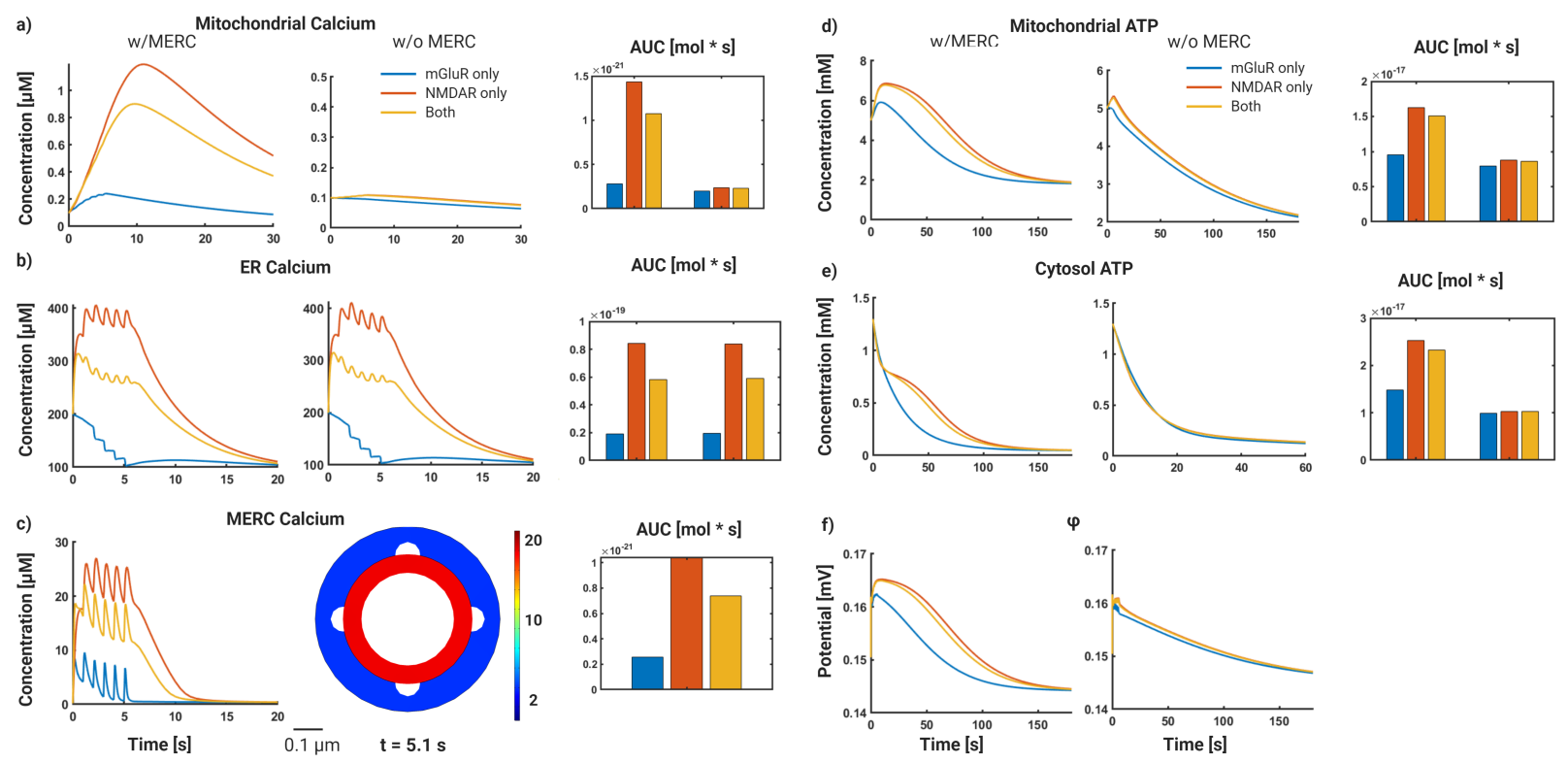

Figure 3: Receptor-based simulations of glutamatergic signaling in spatial calcium model. Finite element simulations of dendritic spines including mitochondria, ER, and mitochondrial-ER contacts(MERC). In all the plots, the blue line is for mGluR only, red for NMDAR only and yellow for both receptors. a) Mitochondrial $\mathrm{Ca}^{2+}$ dynamics in the presence (left) and absence (middle) of MERC. Total $\mathrm{Ca}^{2+}$ calculated using the area under the curve (AUC) (right) b) ER Calcium dynamics with (left) and without MERC (middle); AUC (right). c) Dynamics of MERC $\mathrm{Ca}^{2+}$ in the MERC case only (left); cross section of MERC ring showing a higher concentration of $\mathrm{Ca}^{2+}$ in the MERC ring as opposed to the general cytoplasm (right). d) Dynamics of mitochondrial ATP; with MERC (left), without MERC (middle), AUC (right). e) Dynamics of cytosolic ATP; with MERC (left), without MERC (middle), AUC (right). f) Mitochondrial membrane potential; with MERC (left), without MERC (right). 
increasing mitochondrial size increases the total amount of $\mathrm{Ca}^{2+}$ and ATP produced in the mitochondria. In this model, mitochondrial size is limited by our dendrite segment size, however our results suggest that a larger mitochondria would produce more ATP.

When we look at the cytosolic ATP, we note that smaller mitochondria actually show a decrease in cytosolic ATP. As the mitochondrial size increases, cytosolic ATP increases and at larger mitochondrial sizes the kinetics are prolonged (Figure 4d). This effect of mitochondrial size on cytosolic ATP can be understood as a result of the competition between the boundary fluxes on the mitochondrial membrane for ANT and the hydrolysis rate occurring in the cytosol. As the mitochondrial size increases, the corresponding surface area increases, increasing the flux across the mitochondrial membrane into the cytosol. This change in flux also alters the kinetics of cytosolic ATP. Increasing the length of the mitochondria increases the duration of $\mathrm{Ca}^{2+}$ flux into the mitochondria, especially when there is sufficient MERC to facilitate $\mathrm{Ca}^{2+}$ influx. This increase in the flux shifts the overall $\mathrm{Ca}^{2+}$ concentration, resulting in a longer half-life of mitochondrial ATP and an increase in peak times for cytosolic ATP (Table S4).

Thus, our model predicts that the presence of MERCs alone does not inherently confer an advantage for increased cytosolic ATP for all mitochondrial sizes. Rather, an equilibrium between rapid ATP production in the mitochondria through MERCs and a rapid ATP delivery to the cytosol through ANT are required to balance ATP production and supply in dendrites. ANT also drives ADP transport between the cytosol and mitochondria, which is a necessary substrate for ATP production. For effective ATP availability in the dendrites our model predicts two requirements - production of ATP in the mitochondria and availability of ATP in the cytosol. Production of ATP in the mitochondria depends on MERC area fraction and mitochondrial size. Availability of ATP in the cytosol depends on the flux of ATP from the mitochondria through the ANT and the consumption of ATP through a lumped hydrolysis rate.

\section{Combination of increased mitochondrial size and MERC area fraction buffers ATP produc- tion capacity}

We next investigated the role of MERC and mitochondrial sizes in governing ATP production capacity. Since MERCs provide a direct conduit to increase mitochondrial $\mathrm{Ca}^{2+}$, we varied the area fraction of MERCs with mitochondria of different sizes $(0.3,0.6$, and $1.2 \mu \mathrm{m})$. These lengths are supported by observations that CaCaMKII activity can decrease mitochondrial length over a range of synaptic activity levels [47]. Particularly, we sought to find if increasing MERC area fraction could confer an advantage to smaller mitochondria, compensating for volumetric disadvantage with increased calcium. Experimental evidence indicates that 

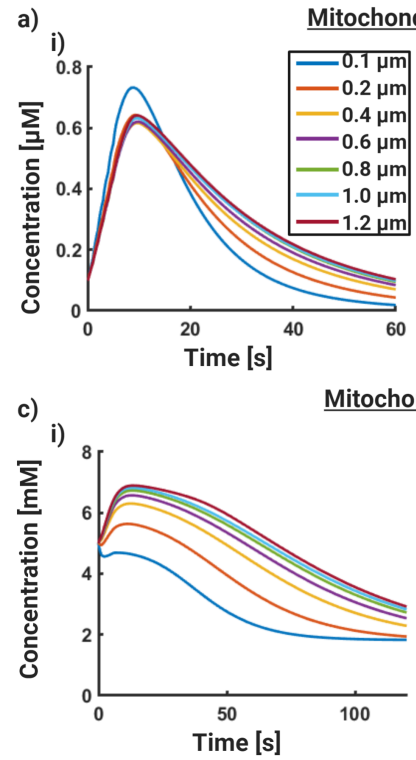

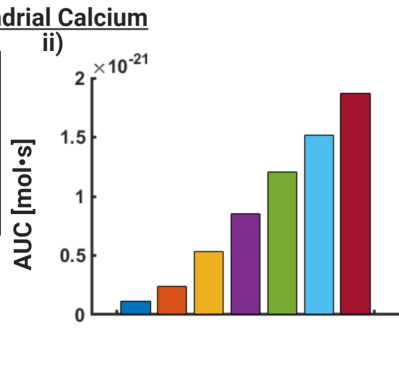

b) i)

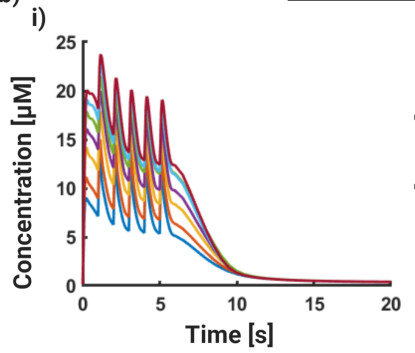

d)

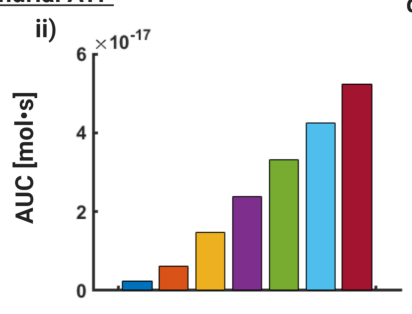

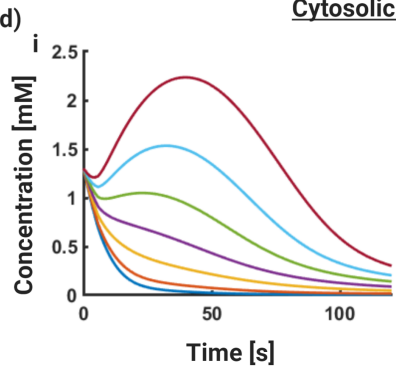

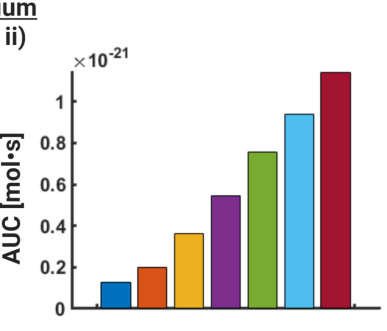

ii)

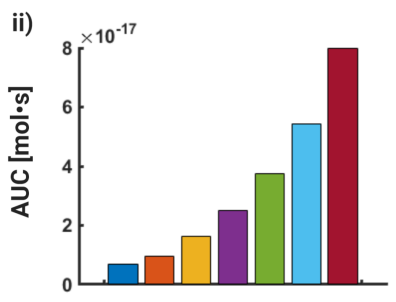

Figure 4: Mitochondrial length increases the magnitude of mitochondrial $\mathrm{Ca}^{2+}$ and ATP In these simulations we increased the length of the mitochondria while keeping the MERC area fraction constant at 0.1. a) (i) Volumetric average dynamics of mitochondrial $\mathrm{Ca}^{2+}$ increases with increasing mitochondrial length; (ii) AUC for mitochondrial $\mathrm{Ca}^{2+}$, integrated over time and mitochondrial volume. b) (i) Volumetric average dynamics of MERC $\mathrm{Ca}^{2+}$; (ii) AUC for MERC $\mathrm{Ca}^{2+}$. c) (i) Volumetric average dynamics of mitochondrial ATP increases with increasing mitochondrial length; (ii) AUC for mitochondrial ATP, integrated over time and mitochondrial volume. d) (i) Volumetric average dynamics of cytosolic $\mathrm{Ca}^{2+}$ increases with increasing mitochondrial length; (ii) AUC for mitochondrial $\mathrm{Ca}^{2+}$, integrated over time and mitochondrial volume. 
MERCs range from 2-20\% of mitochondria surface area depending on the metabolic state and type of cell $[45,48,49]$. We conducted simulations in small $(0.3 \mu \mathrm{m})$, medium $(0.6 \mu \mathrm{m})$, and large mitochondria $(1.2 \mu \mathrm{m})$, with a range of MERC area fractions ranging from $2.5 \%$ to $30 \%$ (Figure 5) for a fixed value of the ATP consumption rate of $200 \mu \mathrm{M} / \mathrm{s}$. We note that, while area fraction ranges have been experimentally quantified, they need not necessarily form a ring-like structure that we model here $[17,48,50,51]$. As shown in Figure S6, the effect of MERCs on cytosolic $\mathrm{Ca}^{2+}$ and $\mathrm{IP}_{3}$ are not dramatically affected by overall size of MERCs.

We observe that increasing the MERC area fraction increases mitochondrial $\mathrm{Ca}^{2+}$ across all sizes but the peak concentration of $\mathrm{Ca}^{2+}$ decreases with increasing mitochondrial size (Figure 5a). This effect is true for both the small and large mitochondria, and the larger mitochondria have higher calcium concentration and total amounts (by virtue of larger volumes). MERC $\mathrm{Ca}^{2+}$ dynamics also show an increase with increasing MERC area fraction for all mitochondrial sizes with a proportionally small increase in the peak $\mathrm{Ca}^{2+}$ (Figure 5b). Mitochondrial ATP production increases with the MERC area fraction within a given mitochondrial size and also increases with larger mitochondrial sizes (Figure 5c). Interestingly, the dynamics of mitochondrial ATP show a faster decay with increasing MERC area fraction for small and medium mitochondria (Figure 5c, i,ii). For the large mitochondria, we observe a second and prolonged peak of mitochondrial ATP as the area fraction of MERC increases (Figure 5c,iii). Thus the combination of MERC area fraction and mitochondrial sizes results in synergistic effects on mitochondrial ATP production. However, looking at the cytosolic ATP dynamics, we note that not all mitochondrial sizes or MERC fractions are conducive to increased ATP availability (Figure 5d). Small mitochondria show practically no increase in cytosolic ATP and are unaffected by MERC area fraction (Figure 5d, i). Medium size mitochondria begin to show some response in that increasing the MERC area fraction prolongs the decay time but no dramatic increase in cytosolic ATP is observed (Figure 5d,ii). Large mitochondria show a strong sensitivity to MERC area fraction - first, large mitochondria show an increase in cytosolic ATP even for small MERC area fractions and second, increasing MERC area fraction in large mitochondria results in a larger ATP concentration in the cytosol (Figure 5iii).

\section{Energy availability at the synapse is a combination of mitochondrial geometry, MERC area fraction, and kinetics of ATP production and consumption}

Finally, we asked the following question: how is the balance between the kinetics of ATP production through F1FO, ATP transport through ANT, and ATP consumption (modeled using a lumped hydrolysis 
a) Mitochondrial Calcium

i)

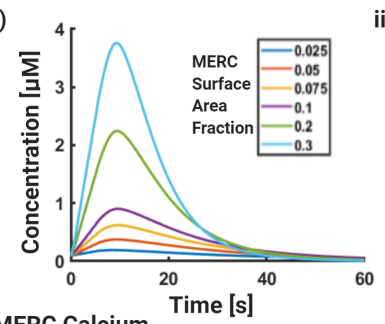

b) MERC Calcium

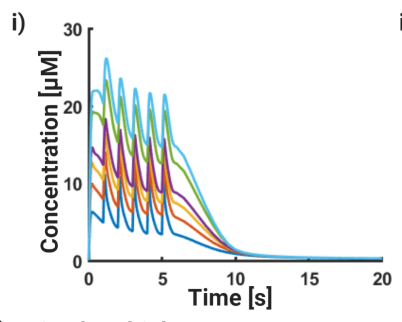

c) Mitochondrial ATP

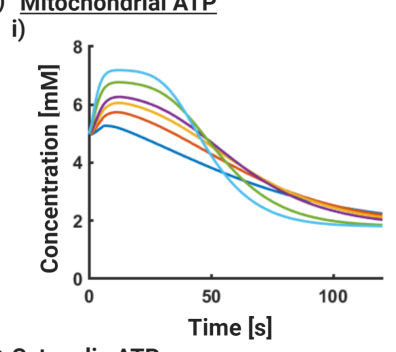

d) Cytosolic ATP

i)

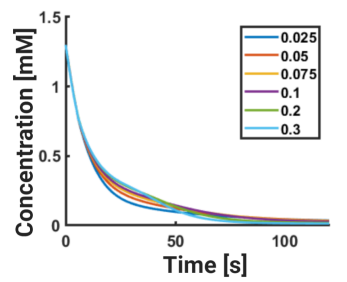

ii)

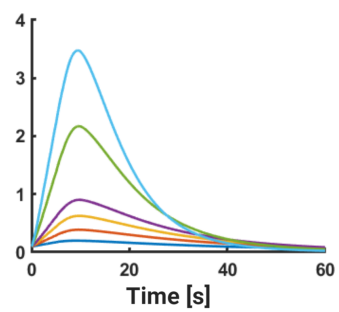

ii)
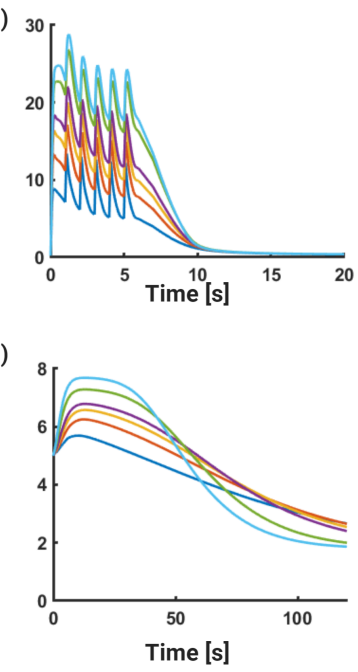

ii)

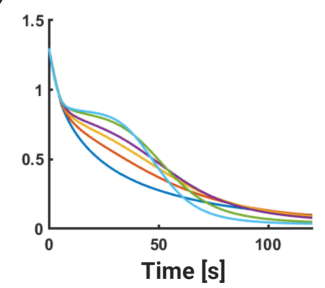

iii)

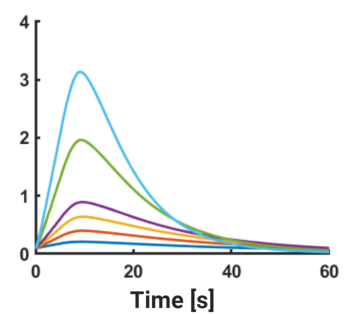

iii)

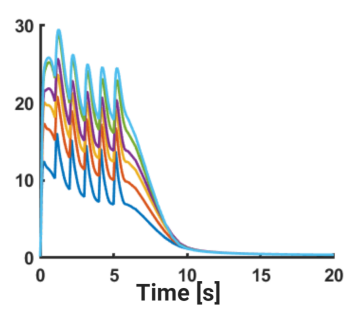

iii)

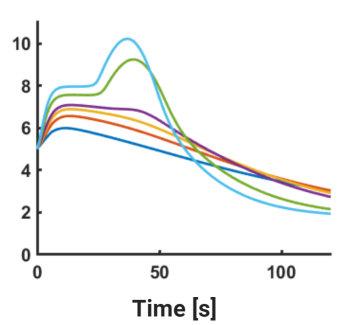

iii)

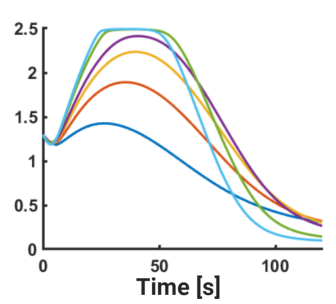

$\underline{\mathrm{AUC}}$
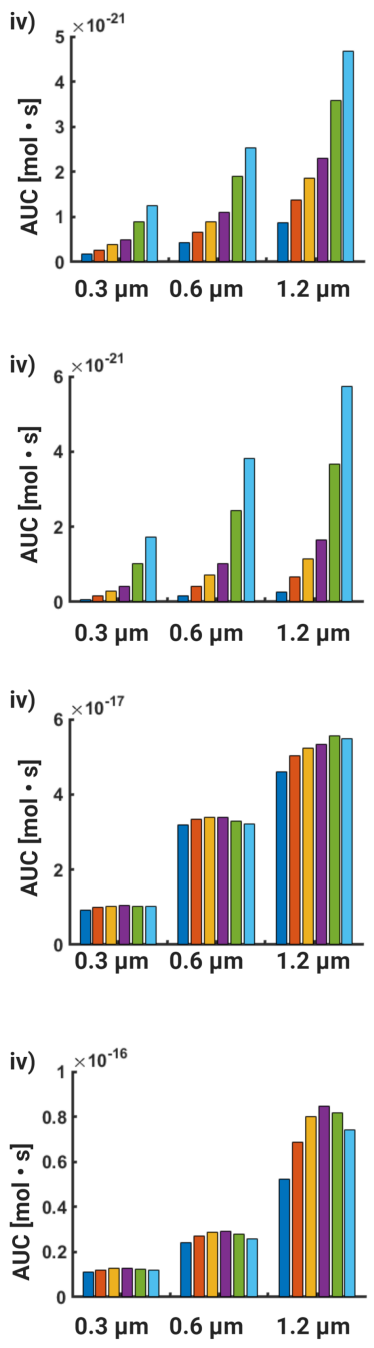

Figure 5: Effect of MERC area fraction on ATP production Here we vary the size of the mitochondria and the area fraction of MERC systematically. Three different mitochondrial sizes $(0.3 \mu \mathrm{m}, 0.6 \mu \mathrm{m}$, and $1.2 \mu \mathrm{m}$ ) and six different MERC area fractions ranging from 0.025 to 0.3 were simulated. a) Mitochondrial $\mathrm{Ca}^{2+}$ dynamics and AUC for the different cases. b) MERC $\mathrm{Ca}^{2+}$ dynamics and AUC for the different cases. c) Mitochondrial ATP dynamics and AUC for the different cases. d) Cytosolic ATP dynamics and AUC for the different cases.

term, $\mathrm{K}_{H Y D}$ ) influenced by mitochondrial size and MERC area fraction? We conducted a series of simulations to map the corresponding phase spaces that hold the answer to this question. For all simulations, we varied mitochondrial size from $0.3-1.2 \mu \mathrm{m}$ and MERC area fraction (defined as the ratio of MERC SA to mitochondria SA) from 0 (no contact) to 0.2. Then, we calculated the AUC for mitochondrial ATP and the 
AUC for cytosolic ATP as our scalar readout.

To investigate the role of the rate of ATP production by the F1,FO ATP synthase, we varied the kinetic parameter $V_{F 1, F O}$ (Figure 6a) (Tables S2 and S5). This variation effectively captures the changes to either the activity of the ATP synthase or the number of ATP synthases. We varied the value of $V_{F 1 F O}$ by two orders of magnitude with respect to our control value of $35 \mu \mathrm{M}$. We found that as we increased $V_{F 1 F O}$, mitochondrial ATP increases with mitochondrial size and MERC area fraction across all the values of $V_{F 1 F O}$. For higher values of $V_{F 1 F O}$, however, we lose the dependence on MERC area fraction obtaining simply a linear dependence on mitochondrial size. In contrast, cytosolic ATP shows a non-monotonic dependence on $V_{F 1 F O}$ for different mitochondrial lengths and MERC SA ratio. For low $V_{F 1 F O}$, cytosolic ATP (Figure 6b) is maximum for large mitochondrial length and for MERC area fractions close to 5\%. As the MERC area fraction increases, the cytosolic ATP decreases. However, for medium values of $V_{F 1 F O}$, cytosolic ATP increases with both mitochondrial length and MERC SA ratio, indicating the increasing rate of ATP synthesis gives high cytosolic ATP for a wide range of MERCs. Further increase in $V_{F 1 F O}$ did not confer any additional advantage on cytosolic ATP; instead the cytosolic ATP was lower for larger mitochondrial lengths and MERC area fraction. Although the AUC captures the total production of ATP, to study the dynamics we plot a selection of points in the phase diagram in Figure S7. We show that the dynamics of ATP production in the mitochondria and ATP availability in the cytosol are dramamtically altered by the values of $V_{F 1 F O}$ and the geometric parameters (Figure S7) suggesting a more complex spatiotemporal regulation.

These results suggest that increasing $V_{F 1 F O}$ increases mitochondrial ATP, but the impact on cytosolic ATP is mixed. This could be because either the model is incomplete because we don't consider the morphology of the inner mitochondrial membrane (see [52] for a detailed inner mitochondrial membrane model) or that the production of ATP is not matched by the transport to the cytosol.

Next, we changed the value of $V_{A N T}$ to study the role of ATP transport across the mitochondrial membrane on the energetic balance between mitochondria and cytosol (Figure 6c). For the low value of $V_{A N T}$, the mitochondrial ATP AUC increases with mitochondrial length and MERC area fraction. The cytosolic ATP AUC also increases for mitochondrial length and MERC area fraction. When $V_{A N T}$ increases, the accessible states for mitochondrial ATP AUC decrease with respect to our geometric parameters (Figure 6c,ii) but remain unchanged for cytosolic ATP(Figure 6d,ii). For high values of $\mathrm{V}_{A N T}$ we see a similar trend as the difference in mitochondria ATP AUC states is nearly abolished (Figure 6c,iii), but has little effect on the cytosolic ATP. Thus, $V_{A N T}$ is a critical parameter in maintaining mitochondrial ATP generation rates across different mitochondrial lengths and MERC area fractions. However, $\mathrm{V}_{A N T}$ does not have an influence on 
the total production of ATP in the system.

Finally, in Figure 6e, we explore the role of the rate of ATP consumption in our system, by varying $k_{h y d}$ Table S5 in the cytosol. This lumped parameter effectively captures the energy consumption in the postsynaptic spine; over $60 \%$ of neuronal energy consumption is consumed during active calcium signaling, which occurs at the PM and ER boundaries [53]. We note that there is limited effect of changing the AUC of mitochondrial ATP across different values of $k_{h y d}$ and MERC SA ratio. There is a linear increase in Mitochondrial ATP AUC with respect to mitochondrial length (Figure 6e). Cytosolic ATP (Figure 6f), on the other hand, decreases with increase in $k_{h y d}$, as expected. For low values of $k_{h y d}$, cytosolic ATP increases with mitochondrial lengths and MERC surface area. As $k_{h y d}$ increases, the consumption of ATP essentially overrides any geometric dependence. Larger mitochondria are able to offset this higher consumption, but as the energy consumption increases the system is unable to reach higher energy states at the current geometry. Thus, our model shows that the metabolic parameters associated with ATP production, transport, and consumption in conjunction with MERC surface area ratio and mitochondrial length are important for ATP availability.

\section{Discussion}

In this study, we have investigated how the presence of MERCs can alter $\mathrm{Ca}^{2+}$ and ATP dynamics in the postsynaptic spine and surrounding areas using computational modeling. Our model predicted that the presence of MERCs can lead into increased mitochondrial calcium transients and increased mitochondrial ATP production consistent with experimental observations [47,50,54]. We also showed that mitochondrial size and MERC area fraction play a critical role in the balance of mitochondrial ATP production and cytosolic ATP by regulating the fluxes into and outside of the mitochondria [47]. And finally, we predict that metabolic parameters associated with mitochondrial function are also important determinants of this balance between energy production and energy availability (Figure 7).

Recently, the mitochondrial dynamics, including fission [47], ROS generation [55], cytochrome c release [56] and MERCs [50] have been implicated in postsynaptic response to different stimuli, particularly in the context of energy requirements of synaptic transmission. Furthermore, the presence of MERCs has been noted as a critical factor in the mitochondrial response and loss of MERCs shows a reduced mitochondrial $\mathrm{Ca}^{2+}$ response in neurons [50]. Dendritic mitochondria can range in size from $1 \mu \mathrm{m}$ to over $10 \mu \mathrm{m}$ in length (median $2.5 \mu \mathrm{m}$ ) $[11,39,47]$ While our spatial model is not to scale (we chose a $1.5 \mu \mathrm{m}$ long dendrite segment for computational ease), we note that our model predicts that dendritic energy availability is 

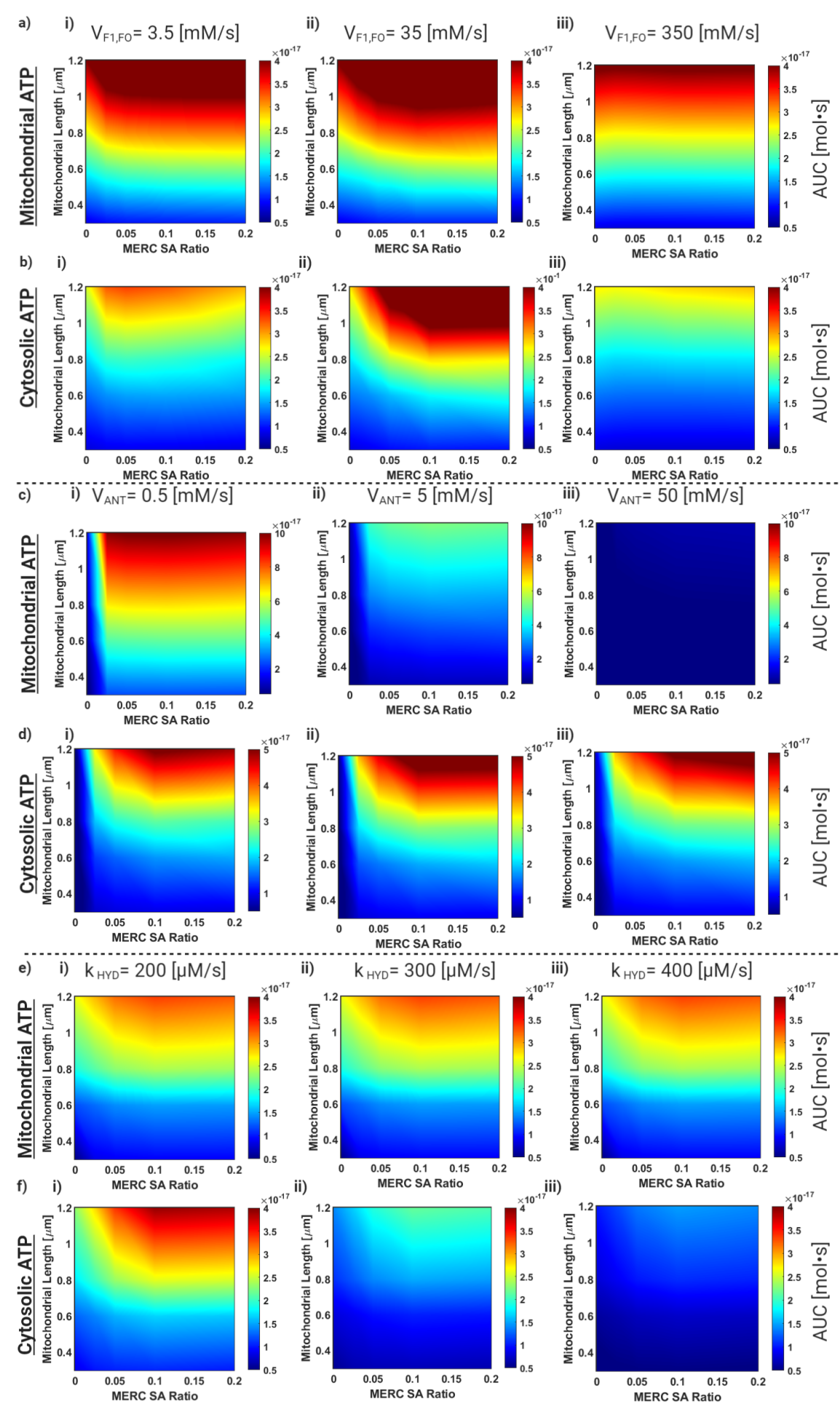

Figure 6: Model overview and discussion of geometric control of metabolic balance a) Cytosolic ATP AUC phase map for i) $V_{F 1, F O}=3.5 \mathrm{mM} / \mathrm{s}$, ii) $V_{F 1, F O}=35 \mathrm{mM} / \mathrm{s}$, and iii) $V_{F 1, F O}=350 \mathrm{mM} / \mathrm{s}$. b) Same as a) for mitochondria ATP. c) Cytosolic ATP AUC phase map for i) $V_{A N T}=0.5 \mathrm{mM} / \mathrm{s}$, ii) $V_{A N T}=5 \mathrm{mM} / \mathrm{s}$, iii) $V_{A N T}=500 \mathrm{mM} / \mathrm{s}$. d) Same as c) for mitochondria ATP. e) Cytosolic ATP AUC phase map for i) $K_{H Y D}=200 \mathrm{mM} / \mathrm{s}$,ii) $K_{H Y D}=300 \mathrm{mM} / \mathrm{s}$, and iii) $K_{H Y D}=400 \mathrm{mM} / \mathrm{s}$. f) Same as e) for mitochondria ATP. 


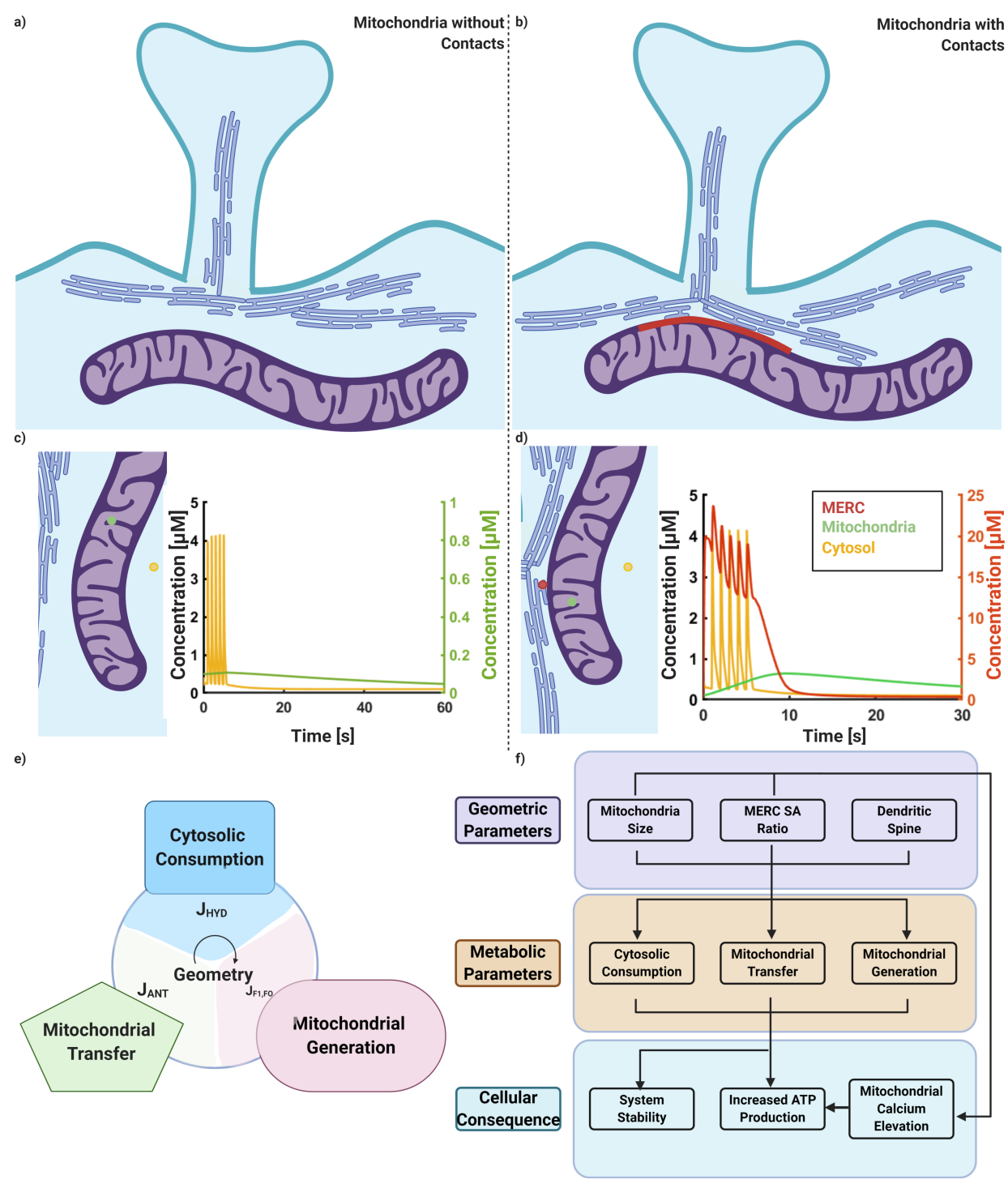

Figure 7: Schematic for the impact of MERCs and geometric regulation of mitochondiral ATP produc-

tion a) Schematic for dendrite with ER not forming contacts with mitochondria. b) Schematic for dendrite with ER forming contacts with mitochondria, highlighted in red c) Calcium profiles for cytosol (in yellow) and mitochondria (in green) for the system without any contact sites. d) Calcium profiles for cytosol (in yellow), mitochondria (in green), and MERC (in red) for system with contact sites . e) Schematic outlining key fluxes that balance ATP in the mitochondria and cytosol explored in Figure 6. f) Flow diagram describing the interconnected influence of cell and organelle geometry on metabolic production with added feedback through calcium signaling. 
directly proportional to mitochondrial length. Divakaruni et al. report that mitochondrial length is linearly proportional to dendritic length [47]; thus the scaling we find here suggests a common size principle for dendritic energy availability. Separately, Hirayabashi and colleagues [50] reported that PDZD8 is required for MERC formation and that the MERC area fraction ranges from 0.02-0.16 in HeLa cells. We note that increasing MERC to mitochondria surface area ratios has influence over the ATP production dynamics as well as the total ATP production. Although MERCs can have a substantial impact on mitochondria ATP generation in contrast to cases without MERC, the MERC area fraction does not show a linear dependence on ATP production as was observed for mitochondrial size. This implies that the benefit of MERCs is closer to that of a switch-like behavior rather than graded behavior. We also show that biochemical parameters associated with metabolism such as ATP production, nucleotide transport, and ATP hydrolysis also play an important role in cytosolic energy availability by allowing for a graded behavior. Thus, we find that there is a unique balance between energetic and biochemical aspects of mitochondrial function in dendrites. This balance is further regulated in cells by $\mathrm{Ca}^{2+}$-dependent mitochondrial fission to complete the feedback loop of signaling and mitochondrial size regulation and will require a deeper understanding of the underlying coupled mechanochemical processes [57].

We note that our model has made some simplifying assumptions to keep the simulations tractable. First, all our geometries are simplified, allowing us to construct a spatial model of multiple organelles. We note that current efforts include using realistic geometries of spines and organelles derived from microscopy to investigate how these play a role in $\mathrm{Ca}^{2+}$ and ATP dynamics $[52,58,59]$. Second, we note that we assume that $\mathrm{Ca}^{2+}$ and ATP are present in large enough amounts to justify a deterministic approach. We recognize that a stochastic approach would likely give rise to insights in limit cases of few molecules or in noisy environments $[37,38]$. Finally, we note that a more complete $\mathrm{Ca}^{2+}$ influx model would account for voltage-gated calcium channel dynamics as well [22,24,60,61]. This is a focus of technical development and ongoing effort in our group.

To summarize, we show using a spatial model of $\mathrm{Ca}^{2+}$ dynamics in dendrites, including the ER, the mitochondria, and MERCs, that spatial organization of organelles and their contacts plays a critical role in determining the downstream response of a spine to a stimulus. This work sets the stage for future investigation on the coupling between biochemical signaling [62], biophysical mechanisms of organelle transport and tethering, and metabolic pathways, giving a glimpse into the complex regulation of neuronal processes. 


\section{Author Contributions}

A. Leung, D. Ohadi, and P. Rangamani conceived the project. D. Ohadi and A. Leung developed spatial model. A. Leung, G. Pekkurnaz and P. Rangamani conducted simulations and data analysis. All authors contributed to writing the manuscript. A. Leung generated all figures.

\section{Acknowledgments}

The authors would like to acknowledge Justin Laughlin and Miriam Bell for their useful discussion and computational resources. Additionally, we acknowledge biorender.com for tools used to generate figures. Finally, this work was supported by the University of California, San Diego Interfaces Graduate Training Program and a San Diego Fellowship, Air Force Office of Scientific Research (AFOSR) Multidisciplinary University Research Initiative (MURI) grant FA9550-18-10051 (A. Leung, P. Rangamani)

\section{Conflicts of Interest}

The authors declare no competing financial interests. 


\section{Supplementary Material}

\section{Mathematical Model Development}

In this section, we discuss the details of the model development, assumptions, parameters, and numerical methods.

\section{Geometry Development}

We built a model with five compartments: the postsynaptic density (PSD), the cytosol, one mitochondria, the ER, and a mitochondria ER Contact region (MERC) (Figure 1).

In our simplified geometry, the dendritic spine is modeled as a sphere attached to the dendritic shaft by the spine neck. The spine neck and the dendritic shaft were modeled as cylinders. (Table S1)

Although the morphology of a spine has been shown to govern the magnitude and stability of calcium transients in dendrites in previous studies [24,31], in this study we simplify complex spine morphology in idealized geometries to focus on the role of mitochondria in neuronal calcium dynamics. While the ER is distributed throughout the cytoplasm, we approximate the ER within the dendritic shaft as long cylinders, keeping volumetric ER to cytoplasm ratios constant. The sizes of these compartments are given in Table S1. While we acknowledge that these simplifications do not capture geometric complexity of a dendrite [48], they allow us to explore the role of spatial organization of the spine in a computational framework.

\section{Neuronal Stimulus Patterns}

While there are a multitude of signaling frequencies and patterns, in this work we focus on a simple periodic stimulus, $1 \mathrm{~Hz}$ for 5 seconds [63] to emulate signaling in an established spine. Mathematically, we approximate glutamate stimulus as discrete pulses in a well-mixed system within COMSOL. Since we are not modeling synaptic vesicles or presynaptic neuron signaling, we apply a series of delta functions temporally spaced according to the desired pulse train (i.e. $1 \mathrm{~Hz}$ stimulus has delta functions spaced 1 seconds apart). Glutamate then decays with a time constant of $1.2 \mathrm{~ms}$ [64]. Key contributing factors to this decay are rapid reabsorbed by the signaling neurons and astrocytic cells as well as diffusion [65].

\section{Calcium Dynamics}

After glutamate binds to receptors on the post-synaptic density (PSD), there is a large calcium influx from the extracellular space and endoplasmic reticulum. This signaling initiates at the dendritic spine and propa- 
gates to the dendritic shaft, where the mitochondria are primarily located. We assume that the extracellular calcium does not deplete and does not alter the flux of synaptic calcium signaling. Extracellular calcium concentrations are typically several orders of magnitude higher than intracellular calcium [66].

The calcium dynamics in our model are based on work done by [25, 27,67], and parameters were adapted and suitably modified to reflect neuronal calcium metabolism.

- Postsynaptic area and Receptor Dynamics: This compartment includes membrane-bound molecules on the postsynaptic area: NMDA receptor (NMDAR), free mGluRs $\left(\mathrm{R}_{2}\right)$, glutamate-bound mGluRs (DIM), phosphorylated mGluRs ( $\mathrm{DIM}_{p}$ ), and second messenger DAG. These molecules localize to the PSD area of spine heads. Calcium enters the spine head through NMDAR. The NMDAR dynamics are described in a multistate model [68]. And the NMDAR-induced calcium influx is modeled as:

$$
J_{N M D A}=I_{N M D A}
$$

where, $I_{N M D A}$ is the current associated with calcium influx. Mathematical descriptions of all terms are expanded in Table S2.

Glutamate binds to the mGluR on the spine head, which induces $\mathrm{G}_{q \alpha}$ to separate from $\mathrm{G}_{q \beta \gamma}$ and activate $\mathrm{PLC}_{\beta}$ enzyme. $\mathrm{PLC}_{\beta}$ enzyme cleaves $\mathrm{PIP}_{2}$ (a membrane phospholipid) and generates DAG and $\mathrm{IP}_{3}$. While DAG remains on the membrane and activates PKC, which then may phosphorylates mGluR, phosphotases dephosphorylate mGluR. This phosphorylation can inhibit the dimerization of $\mathrm{mGluR} \mathrm{IP}_{3}$ diffuses through the cytosol and initiates $\mathrm{Ca}^{2+}$ release from internal stores by binding to receptors $\left(\mathrm{IP}_{3} \mathrm{R}\right)$ on the ER membrane. $\mathrm{IP}_{3}$ in the cytosol is degraded to $\mathrm{IP}_{2}$ and $\mathrm{IP}_{4}$. The degradation is assumed to recycle phospholipids such that $\mathrm{IP}_{3}$ production is not rate limited by $\mathrm{PIP}_{2}$ availability. Although not modeled here, $\mathrm{Ca}^{2+}$ can also be released from ER by Ryanodine receptors and conversely can be actively transported back into the ER by Sarcoplasmic/endoplasmic reticulum calcium-ATPase (SERCA) on the ER membrane.

Equations associated with the mGluR cascade are included in Table S2. The calcium flux is modeled as a boundary condition on the endoplasmic reticulum membrane, in contrast to the NMDAR on the PSD.

- Cytosolic Calcium Dynamics: $\mathrm{Ca}^{2+}$ transients that accumulate due to influx at the PSD are able to diffuse throughout the cytosol. While we do not explicitly model calcium buffers, buffering capacity is included through boundary conditions between compartments. Cytosolic $\mathrm{Ca}^{2+}$ dynamics in $3 \mathrm{D}$ is 
defined as:

$$
\frac{\partial\left[C a_{c}^{2+}\right]}{\partial t}=D_{C a^{2+}} \nabla^{2}\left[C a_{c}^{2+}\right]-J_{B u f f}
$$

where $D_{\mathrm{Ca}^{2+}}$ is diffusion coefficient and $\nabla^{2}$ is Laplacian operator in $3 \mathrm{D}$. The boundary condition for $\mathrm{Ca}^{2+}$ influx through NMDAR in postsynaptic area and $\mathrm{Ca}^{2+}$ efflux from cytosol is defined as,

$$
\left.D_{C a^{2+}}\left(\mathbf{n} . \nabla\left[C a_{c}^{2+}\right]\right)\right|_{P S D}=\left(J_{N M D A}-J_{e f f}\right) m_{p}
$$

where $m_{p}$ is a geometric factor for fluxes on the boundary and is defined as the ratio of the cytosol volume to the postsynaptic surface area. $\mathbf{J}_{N M D A}$ is defined as the flux as a result of the NMDA receptor activity. $\mathrm{J}_{\text {eff }}$ is defined as the activity as a result of plasma membrane calcium ATP-ase.

The boundary fluxes on ER for cytosolic $\mathrm{Ca}^{2+}$ are defined as:

$$
\left.D_{C a^{2+}}\left(\mathbf{n} . \nabla\left[C a_{c}^{2+}\right]\right)\right|_{E R}=b_{c}\left(\alpha J_{I P_{3}}+\alpha J_{R y n}-J_{S E R C A}\right) m_{e}
$$

in which $\mathrm{b}_{c}$ is the buffering capacity of cytosol and $\alpha$ is ER to cytosol volume ratio. $J_{I P_{3} R}$ is the $\mathrm{Ca}^{2+}$ flux from the ER into the cytosol through the $\mathrm{IP}_{3}$ receptor, $J_{R Y R}$ is the $\mathrm{Ca}^{2+}$ flux from the ER into the cytosol through the Ryanodine receptor, $J_{S E R C A}$ is the $\mathrm{Ca}^{2+}$ flux from the cytosol into the ER through the SERCA ATPase pumps, and $m_{e}$ is a geometric factor for fluxes on the boundary and is defined as the ratio of the cytosolic volume to the ER surface area. $J_{I P_{3} R}$ and $J_{R Y R}$ are defined with respect to ER volume and $J_{S E R C A}$ is defined with respect to the cytosol volume. The mitochondrial boundary fluxes for cytosolic $\mathrm{Ca}^{2+}$ are defined as:

$$
\left.D_{C a^{2+}}\left(\mathbf{n} . \nabla\left[C a_{c}^{2+}\right]\right)\right|_{M i t}=b_{c}\left(-\beta J_{M C U}+\beta J_{N C X}-\beta J_{m P T P}\right)
$$

in which $\beta$ is mitochondria to cytosol volume ratio. $J_{M C U}$ is $\mathrm{Ca}^{2+}$ flux from the cytosol into the mitochondrion through the MCU channel, $J_{N C X}$ is $\mathrm{Ca}^{2+}$ flux from the mitochondrion into the cytosol through $\mathrm{Na}^{+} / \mathrm{Ca}^{2+}$ exchanger, and $J_{m P T P}$ is $\mathrm{Ca}^{2+}$ bidirectional flux of the mitochondrial Permeability Transition Pore. All of the fluxes within Eq. 5 are defined with respect to mitochondrion volume.

- Calcium buffering: Calcium buffering is included both explicitly and implicitly. The explicit calcium buffers as diffusive buffering molecules and immobile buffers are included as boundary conditions at the plasma membrane.

$$
\frac{\partial B}{\partial t}=D_{B} \nabla^{2} B-J_{B u f f}
$$




$$
\left.D_{C a^{2+}}\left(\mathbf{n} . \nabla\left[C a_{c}^{2+}\right]\right)\right|_{P M}=b_{c}\left(-\beta J_{B u f f, I M}\right)
$$

Implicitly, we consider buffering terms within the boundary conditions between compartments. These buffering terms are denoted as $b_{m}, b_{c}, b_{e r}$ and $\mathrm{b}_{M E R C}$.

- Mitochondrial Calcium Dynamics: The spatiotemporal dynamics of mitochondrial $\mathrm{Ca}^{2+}$ is given by

$$
\frac{\partial\left[C a_{m}^{2+}\right]}{\partial t}=D_{C a^{2+}} \nabla^{2}\left[C a_{m}^{2+}\right]
$$

The boundary fluxes on mitochondrion surface are defined as:

$$
\left.D_{C a^{2+}}\left(\mathbf{n} . \nabla\left[C a_{m}^{2+}\right]\right)\right|_{C y t}=b_{m}\left(J_{M C U}-J_{N C X}+J_{m P T P}\right)
$$

in which $\mathrm{b}_{m}$ is the buffering capacity of $\mathrm{Ca}^{2+}$ in the mitochondrion.

We do not consider the distinction between the outer and inner mitochondrial membrane. Voltagedependent anion channels transport calcium highly efficiently during neuronal activation. Thus, we may assume, as done in [26] [25], that the calcium concentrations in the cytosol and outer mitochondrial calcium approach a rapid equilibrium. The inner mitochondrial membrane calcium fluxes are dependent solely on the cytosolic calcium. The same applies to fluxes regarding the mitochondria ER contact microdomain, which is represented in this model as a separate compartment.

- ER Calcium Dynamics: The only variable in ER compartment is $\mathrm{Ca}_{e}^{2+} \cdot \mathrm{Ca}^{2+}$ in the ER is defined as:

$$
\frac{\partial\left[C a_{e}^{2+}\right]}{\partial t}=D_{C a^{2+}} \nabla^{2}\left[C a_{e}^{2+}\right]
$$

The boundary fluxes on ER are defined as:

$$
\begin{gathered}
\left.D_{C a^{2+}}\left(\mathbf{n} . \nabla\left[C a_{e}^{2+}\right]\right)\right|_{C y t}=b_{e}\left(-J_{I P_{3} R}-J_{R Y R}+\frac{1}{\alpha} J_{S E R C A}\right) \\
\left.D_{C a^{2+}}\left(\mathbf{n} . \nabla\left[C a_{M E R C}^{2+}\right]\right)\right|_{M E R C}=b_{e}\left(-J_{I P_{3} R}-J_{R Y R}+\frac{1}{\alpha} J_{S E R C A}\right)
\end{gathered}
$$

in which $b_{e}$ is the buffering capacity of ER. While the endoplasmic reticulum is a sprawling network of interconnected membranes containing a wide range of proteins and ribosomes, we simplify this network into a series of tubes that span the length of the model dendritic shaft.

\section{- Mito-ER Contacts:}

The ER is known to closely contact the mitochondria and form junctions known as mitocondria-ER Contacts (henceforth referred to as MERC) [17] [46]. This close proximity, which can be as small as 
$10 \mathrm{~nm}$ and is filled with actin [69],calcium binding proteins [18], and pathways that inhibit calcium reuptake [46]. Therefore we model the calcium dynamics in these regions as a separate microdomain from the cytosol. In order to activate $\mathrm{IP}_{3} \mathrm{R}$ on the ER surface facing this microdomain, $\mathrm{IP}_{3}$ must also be present. Thus there are 2 main components within this microdomain, $\mathrm{Ca}^{2+}$ and $\mathrm{IP}_{3}$.

We do not model the molecular composition of the MERC microdomain that control the influx and efflux of $\mathrm{Ca}^{2+}$. Rather, we use the diffusive fluxes at the MERC boundaries as lumped parameters to reflect the different protein distributions across the MERC boundaries.

We model the geometry of this microdomain as a shell that surrounds the circumference of the mitochondria and connects to the endoplasmic reticulum. This shape was chosen to reflect EM images of MERCs and to efficiently model the portion of ER in contact with the mitochondria.

The calcium concentration within this compartment is given by:

$$
\frac{\partial\left[C a_{M E R C}^{2+}\right]}{\partial t}=D_{C a^{2+}} \nabla^{2}\left[C a_{M E R C}^{2+}\right]
$$

And the boundary conditions for the interface between the MERC and the ER, Mitochondria, and cytosol are given as follows:

$$
\begin{gathered}
\left.D_{C a^{2+}}\left(\mathbf{n} . \nabla\left[C a_{M E R C}^{2+}\right]\right)\right|_{C y t}=b_{E-C}\left(J_{C a, D i f f}\right) \\
\left.D_{C a^{2+}}\left(\mathbf{n} . \nabla\left[C a_{M E R C}^{2+}\right]\right)\right|_{E R}=b_{e}\left(-J_{I P_{3} R}-J_{R Y R}+\frac{1}{\alpha} J_{S E R C A}\right) \\
\left.D_{C a^{2+}}\left(\mathbf{n} . \nabla\left[C a_{M E R C}^{2+}\right]\right)\right|_{M i t o}=f m\left(-J_{M} C U_{M} E R C+J_{N} C X_{M} E R M C\right) b_{M M}
\end{gathered}
$$

\section{Energetic considerations in the model}

In this model, we focus on the impact on calcium on mitochondrial ATP production, as done in [25]. Our metabolic model focuses on NAD and NADH within the mitochondria, and ATP and ADP in both the mitochondria and cytosol. To avoid modeling the complex spatial patterns of the inner mitochondrial membrane, we assume that ADP conversion into ATP is a volumetric reaction within the mitochondria. Redox and mitochondrial potential are likewise modeled as volumetric terms within the mitochondria.

$$
\frac{\partial\left[A T P_{m}\right]}{\partial t}=D_{A T P} \nabla^{2}\left[A T P_{m}\right]+J_{F 1 F O}
$$


ATP and ADP are exchanged through the Adenine Nucleotide Transporter (ANT), which is modeled as a boundary condition.

$$
J_{A N T}=V_{A N T} \frac{\left(1-R_{c} R_{m} e^{-\frac{F \Delta \Psi}{R T}}\right)}{\left(1+R_{c} e^{-f \frac{F \Delta \Psi}{R T}}\right)\left(1+R_{m}\right)}
$$

After transfer into the cytosol, ATP freely diffuses and is hydrolyzed by unspecified reactions in the cytosol.

\section{Numerical Methods}

The $\mathrm{Ca}^{2+}$ concentrations and other variables concentrations in all compartments are high enough to be modeled through a deterministic approach. Simulations were conducted using the commercially available finite-element software COMSOL Multiphysics 5.4 [70]. In order to solve our system of partial differential equations, we used time-dependent general partial differential equations and general boundary partial differential equations modules [70]. Starting with a coarse and unstructured mesh, we decreased the mesh size until we obtained the same results when using the maximum mesh size. COMSOL was allowed to optimize the element sizes through the "physics-controlled mesh" option. The linear system was solved directly by using the PARADISO solver on a Linux-based compute cluster. Newton's method (nonlinear method) was used to linearize the system. Time integration was performed using a backward differentiation formula (BDF) with both adaptive order and adaptive step sizes. All COMSOL source files will be available on Rangamani lab Github page upon publication.

\section{Supplementary tables}


Table S1: Model geometry

\begin{tabular}{|c|c|c|c|c|c|}
\hline Organelle & Shape & Length $(\mu \mathrm{m})$ & Diameter $(\mu \mathrm{m})$ & Surface area $\left(\mu \mathrm{m}^{2}\right)$ & Volume $\left(\mu \mathrm{m}^{3}\right)$ \\
\hline Dendrite & cylinder & 1.6 & 0.6 & & \\
\hline Mitochondrion & cylinder & 0.6 & 0.3 & & \\
\hline Tubule mitochondrion & cylinder & 1.2 & 0.21 & & \\
\hline Each of 4 tubule ER & cylinder & 1.5 & 0.08 & & \\
\hline Spine 1 (head) & sphere & - & 0.6 & & $0.058[71]$ \\
\hline Spine 1 (neck) & cylinder & 0.4 & 0.2 & & \\
\hline Spine 1 (PSD) & spherical cap & & & $0.132[72]$ & \\
\hline Spine 2 (head) & sphere & - & 0.4 & & $0.028[71]$ \\
\hline Spine 2 (neck) & cylinder & 0.5 & 0.1 & & \\
\hline Spine 2 (PSD) & spherical cap & & & $0.055[72]$ & \\
\hline Spine apparatus (head) & sphere & - & 0.34 & & \\
\hline Spine apparatus (neck) & cylinder & 0.5 & 0.06 & & \\
\hline Spine apparatus (base) & cylinder & 1.5 & 0.08 & & \\
\hline Distance between spine 1 and spine 2 & - & 1 & - & & \\
\hline \multirow[t]{2}{*}{ Distance between the ER and mitochondria } & - & $0.050[41]$ & - & & \\
\hline & - & $0.030[73]$ & - & & \\
\hline
\end{tabular}

Table S2: Fluxes and Description

\begin{tabular}{|c|c|c|}
\hline Flux Equation & Involved Species & Reference \\
\hline \multicolumn{3}{|l|}{ Module 1: Stimulus and Receptors } \\
\hline \multicolumn{3}{|l|}{ ligand(glutamate)-receptor (mGluR) binding rate } \\
\hline$J_{L R}=-k_{b} R_{2} G^{2}+k_{u} D I M$ & $\mathrm{R}_{2}, \mathrm{G}, \mathrm{DIM}$ & {$[74]$} \\
\hline \multicolumn{3}{|l|}{ PKC-mediated phosphorylation and phosphatase dephosphorylation of mGluR dimers } \\
\hline $\begin{array}{l}J_{D I M}=V_{P} \frac{D I M_{p}}{K_{m P}+D I M_{p}}-V_{P K C} \frac{P K C \cdot D I M}{K_{m P K C}+D I M} \\
\text { phosphorylated mGluR dimers }\end{array}$ & DIM, PKC & {$[74]$} \\
\hline \multicolumn{3}{|l|}{$D I M_{p}=\frac{R_{t o t}-\sqrt{K_{d i m} R_{2}}-2 R_{2}-2 D I M}{2}$} \\
\hline \multicolumn{3}{|l|}{ Change in DAG-sensitive fraction of active PKC } \\
\hline$\frac{d P K C}{d t}=k_{a P K C} \frac{D A G}{K_{m D A G}+D A G}(1-P K C)-k_{d P K C} P K C$ & DAG, PKC & {$[74]$} \\
\hline \multicolumn{3}{|l|}{$\mathrm{IP}_{3}$ synthesis by PLC } \\
\hline$J_{I P_{3}}=k_{P L C_{I}} D I M$ & DIM, IP $_{3}$ & {$[74] \dagger$} \\
\hline \multicolumn{3}{|l|}{ DAG synthesis by PLC and DAG degradation by lipase and kinase } \\
\hline$J_{D A G}=k_{P L C_{D}} D I M-V_{D A G} \frac{D A G}{K_{m D A G}+D A G}$ & DIM, DAG & [74] \\
\hline \multicolumn{3}{|l|}{$\mathrm{Ca}^{2+}$ influx from NMDA receptor } \\
\hline $\begin{array}{ll}p_{o} a, & 0<t<t_{o} \\
0, & t>t_{o}\end{array}$ & & [75] \\
\hline \multicolumn{2}{|l|}{ Increase in $\mathrm{Ca}^{2+}$ concentration per unit time during the NMDA channel opening $a=\frac{I_{C a}{ }^{2+}}{2 e V_{c y t} N_{a}}$} & {$[75]$} \\
\hline \multicolumn{3}{|l|}{ Module 2: Calcium Dynamics } \\
\hline & Con & n next page \\
\hline
\end{tabular}




\begin{tabular}{|c|c|c|}
\hline Flux Equation & Involved Species & Reference \\
\hline \multicolumn{3}{|c|}{$\mathrm{Ca}^{2+}$ current from the NMDA receptor (assuming reversalpotential $=0$ ) } \\
\hline$I_{C a^{2+}}=\frac{-4\left(P_{C a} / P_{M}\right)\left(\left[C a_{e x t}^{2+}\right] /\left[M^{+}\right]\right) \gamma R T}{2 F}$ & & [75] \\
\hline \multicolumn{3}{|l|}{$\mathrm{Ca}^{2+}$ efflux from the cytosol to the extracellular medium } \\
\hline$J_{e f f}=k_{e x} C a_{c}^{2+}$ & $\mathrm{Ca}_{c}^{2+}$ & {$[75]$} \\
\hline \multicolumn{3}{|l|}{$\mathrm{Ca}^{2+}$ flux from the ER into the cytosol through the $\mathrm{IP}_{3}$ receptor } \\
\hline$J_{I P_{3} R}=\left(k_{I P} b+k_{I P} I P R_{a c t}\right)\left(C a_{e}^{2+}-C a_{c}^{2+}\right)$ & $\mathrm{Ca}_{c}^{2+}, \mathrm{Ca}_{e}^{2+}$ & {$[27]$} \\
\hline \multicolumn{3}{|l|}{ Fraction of active $\mathrm{IP}_{3}$ receptors } \\
\hline$I P R_{a c t}=\left(1-R_{i}\right) \frac{I P_{3}^{2}}{K_{I P}^{2}+I P_{3}^{2}} \frac{C a_{c}^{n a}}{K_{d a}^{n a}+C a_{c}^{n a}}$ & $\mathrm{Ca}_{c}^{2+}$ & {$[27]$} \\
\hline \multicolumn{3}{|l|}{ Change in the fraction of inactive $\mathrm{IP}_{3}$ receptors } \\
\hline$\frac{d R_{i}}{d t}=k_{a i}\left(1-R_{i}\right) \frac{C a_{c}^{n i}}{1+\left(\frac{C a_{c}}{K_{d a}}\right)^{n a}}-k_{d i} R_{i}$ & $\mathrm{Ca}_{c}^{2+}$ & {$[27]$} \\
\hline \multicolumn{3}{|c|}{$\mathrm{Ca}^{2+}$ flux from the ER into the cytosol through the Ryanodine receptor } \\
\hline$J_{R Y R}=\left(V_{R Y R} P_{O}+V_{l e a k}\right)\left(C a_{e}^{2+}-C a_{c}^{2+}\right)$ & $\mathrm{Ca}_{c}^{2+}, \mathrm{Ca}_{e}^{2+}$ & {$[76]$} \\
\hline \multicolumn{3}{|c|}{ Open probability of Ryanodine receptor on the slow (second) timescale } \\
\hline$P_{O}=\frac{\omega\left(1+C a_{c}^{3} / K_{b}^{3}\right)}{K_{a}^{4} / C a_{c}^{4}+1+C a_{c}^{3} / K_{b}^{3}}$ & $\mathrm{Ca}_{c}^{2+}$ & {$[76]$} \\
\hline \multicolumn{3}{|c|}{ Fraction of channels not in $\mathrm{Ca}^{2+}$-independent closed state $\left(\omega=1-P_{C_{2}}\right)$} \\
\hline$\tau \frac{d w}{d t}=\omega_{\infty}-\omega$ & $\mathrm{Ca}_{c}^{2+}$ & {$[76]$} \\
\hline \multicolumn{3}{|l|}{ Equilibrium value of $\omega$ and it's relaxation time } \\
\hline$\omega_{\infty}=\frac{1+K_{a}^{4} / C a_{c}^{4}+C a_{c}^{3} / K_{b}^{3}}{1+\left(1 / K_{c}\right)+K_{a}^{4} / C a_{c}^{4}+C a_{c}^{3} / K_{b}^{3}} \quad \tau=\frac{\omega_{\infty}}{K_{d}}$ & $\mathrm{Ca}_{c}^{2+}$ & [76] \\
\hline \multicolumn{3}{|c|}{$\mathrm{Ca}^{2+}$ flux from the cytosol into the ER through the SERCA ATPase pumps } \\
\hline$J_{S E R C A}=V_{S E R C A} \frac{C a_{c}^{2}}{K_{p}^{2}+C a_{c}^{2}} \frac{A T P_{c}}{K_{d s}+A T P_{c}}$ & $\mathrm{Ca}_{c}^{2+}, \mathrm{ATP}_{c}$ & {$[27]$} \\
\hline \multicolumn{3}{|c|}{$J_{M C U}=V_{M C U} \frac{\frac{C a_{c}}{K_{\operatorname{trans}}}\left(1+\frac{C a_{c}}{K_{\text {trans }}}\right)^{3}}{\left(1+\frac{C a_{c}}{K_{\text {trans }}}\right)^{4}+\frac{L}{\left(1+\frac{C a_{c}}{K_{\text {act }}}\right)^{n a u}}} e^{p_{1} \Delta \Psi}$} \\
\hline \multicolumn{3}{|c|}{$\mathrm{Ca}^{2+}$ flux from the mitochondrion into the cytosol through the NCX } \\
\hline$J_{N C X}=V_{N C X}\left(\frac{C a_{m}}{C a_{c}}\right) e^{p_{2} \Delta \Psi}$ & $\mathrm{Ca}_{c}^{2+}, \mathrm{Ca}_{m}^{2+}, \Delta \Psi$ & {$[25]$} \\
\hline \multicolumn{3}{|c|}{$\mathrm{Ca}^{2+}$ bidirectional flux of the mitochondrial Permeability Transition Pore } \\
\hline$J_{m P T P}=k_{m P T P}\left(C a_{c}-C a_{m}\right) e^{p_{3} \Delta \Psi}$ & $\mathrm{Ca}_{c}^{2+}, \mathrm{Ca}_{m}^{2+}, \Delta \Psi$ & {$[27]$} \\
\hline \multicolumn{3}{|l|}{ Buffering Equation } \\
\hline$J_{B u f f}=k_{B u f f}\left(C a_{c} B-k_{e q, B u f f} B C a\right)$ & $C a_{C}, B, B C a$ & {$[24]$} \\
\hline
\end{tabular}

Module 3: Energetics

Glycolytic pathway, Krebs cycle, and PDH-catalysed reaction rate

$J_{P D H}=k_{G L Y}\left(\frac{C a_{m}}{K_{m C a}+C a_{m}}\right)\left(\frac{1}{K_{m N A D}+\frac{N A D H}{N A D^{+}}}\right) \quad \mathrm{Ca}_{m}^{2+}, \mathrm{NADH}^{2} \mathrm{NAD}^{+}$

$\mathrm{Ca}^{2+}$ regulation of the $\mathrm{Ca}^{2+}$ binding mitochondrial AGCs

$J_{A G C}=V_{A G C}\left(\frac{C a_{c}}{K_{A G C}+C a_{c}}\right)\left(\frac{K_{i C a}}{K_{i C a}+C a_{m}}\right) \quad \mathrm{Ca}_{c}^{2+}, \mathrm{Ca}_{m}^{2+}$

$\mathrm{NADH}$ oxidation in the ETC and proton extrusion from mitochondria

$J_{O}=V_{O}\left(\frac{N A D H}{K_{O}+N A D H}\right) \frac{1}{1+e^{\frac{\Delta \Psi-p_{4}}{p_{5}}}}$

$\mathrm{NADH}, \Delta \Psi$

ANT activity rate

$J_{A N T}=V_{A N T} \frac{\left(1-R_{c} R_{m} e^{-\frac{F \Delta \Psi}{R T}}\right)}{\left(1+R_{c} e^{-f \frac{F \Delta T}{R T}}\right)\left(1+R_{m}\right)}$

$\mathrm{R}_{c}, \mathrm{R}_{m}, \Delta \Psi$

$R_{c}=\frac{\alpha_{c} A T P_{c}}{A D P_{c}}, R_{m}=\frac{A D P_{m}}{\alpha_{m} A T P_{m}}$

$\mathrm{ATP}_{c}, \mathrm{ADP}_{c}, \mathrm{ADP}_{m}, \mathrm{ATP}_{m}$ ATP synthesis/ADP phosphorylation rate by the F1FO ATPases 
bioRxiv preprint doi: https://doi.org/10.1101/2020.09.12.294827; this version posted September 13, 2020. The copyright holder for this preprint (which was not certified by peer review) is the author/funder. All rights reserved. No reuse allowed without permission.

Table S2 - continued from previous page

\begin{tabular}{lcc}
\hline \multicolumn{1}{c}{ Flux Equation } & Involved Species & Reference \\
\hline$J_{F 1 F O}=V_{F 1 F O} \frac{1}{1+e^{\frac{p_{6}-\Delta \Psi}{p_{7}}}\left(\frac{K_{i A T P}}{K_{i A T P}+A T P_{m}}\right)}$ & $\mathrm{ATP}_{m}, \Delta \Psi$ & {$[25]$} \\
ATP consumption rate linked with $\mathrm{Ca}^{2+}$ activity in the cytosol & & \\
$J_{H Y D}=V_{H Y D}\left(\frac{A T P_{c}}{K_{m H Y}+A T P_{c}}\right)$ & $\mathrm{ATP}_{c}, \mathrm{Ca}_{c}^{2+}$ & {$[27]$} \\
Proton leak across the inner mitochondrial membrane & & $\Delta \Psi$ \\
$J_{H l e a k}=p_{8} \Delta \Psi+p_{9}$ & {$[25]$} \\
\hline
\end{tabular}




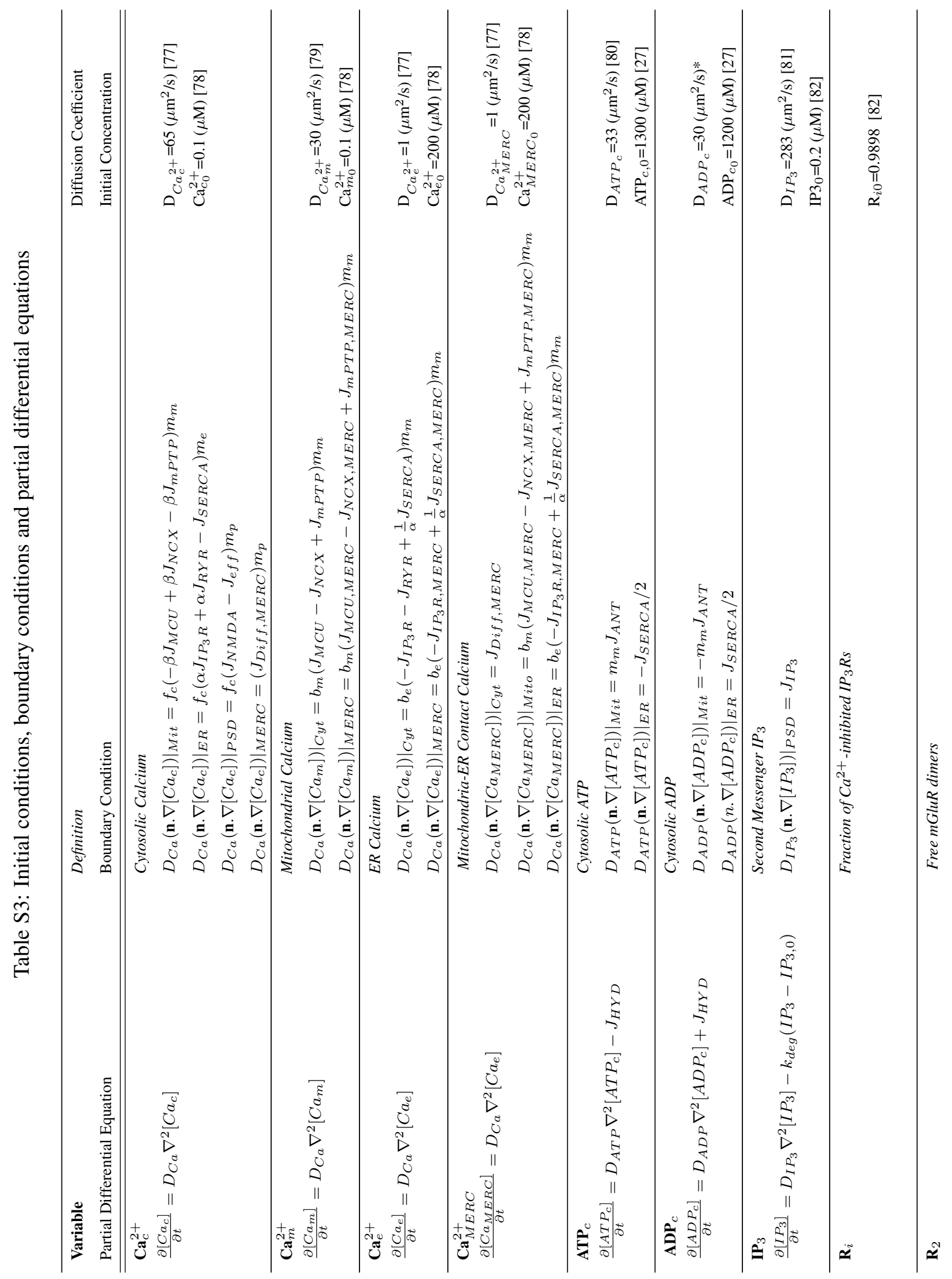




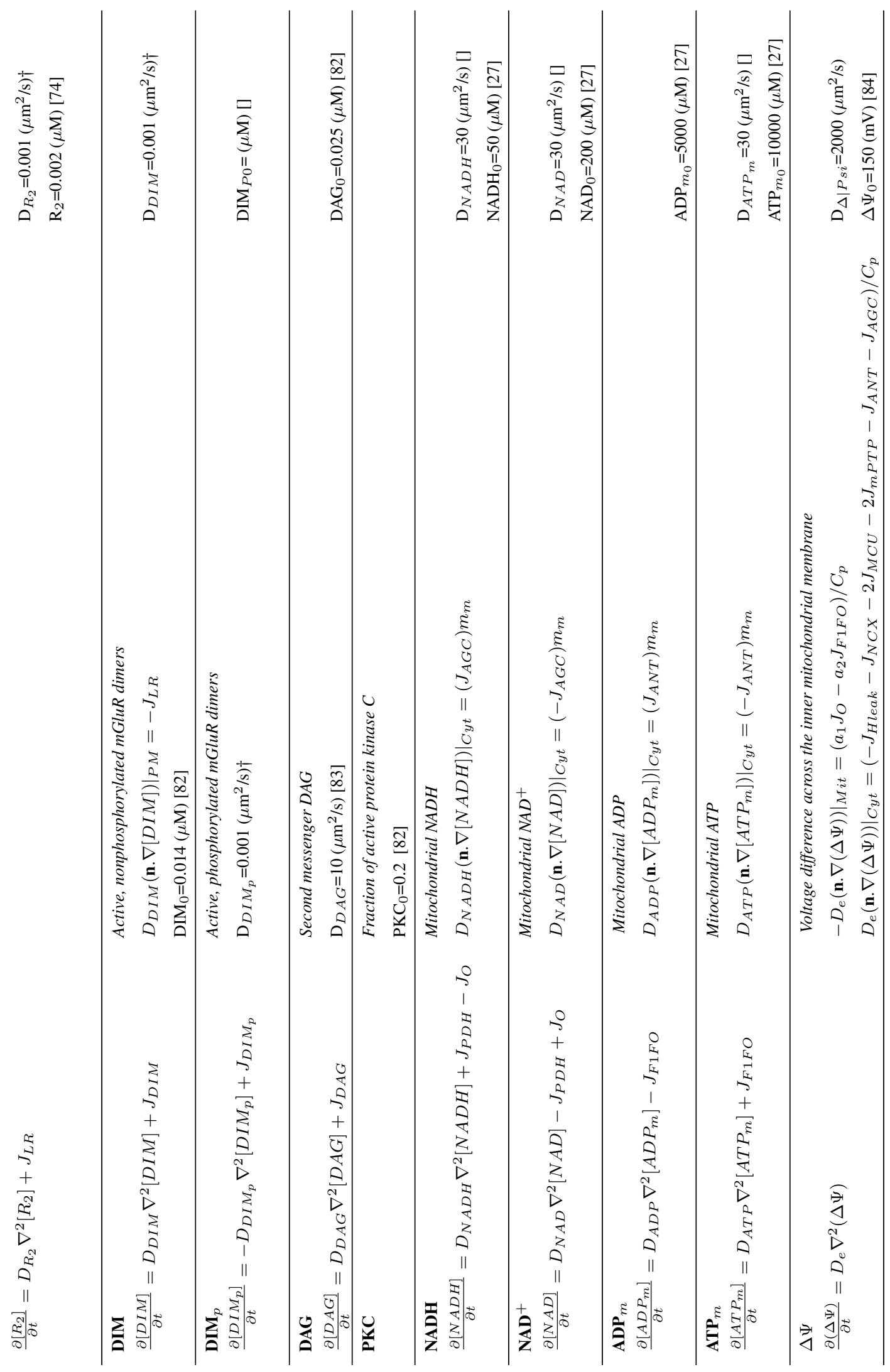


Table S4: Peak Times for Figure 4

\begin{tabular}{ccc}
\hline Mitochondrial Length $[\mu \mathrm{m}]$ & $\mathrm{ATP}_{M}$ Peak Time [s] & $\mathrm{ATP}_{C}$ Peak Time [s] \\
\hline 0.1 & 6.1 & 0 \\
0.2 & 10.2 & 0 \\
0.4 & 11.7 & 0 \\
0.6 & 12.0 & 0 \\
0.8 & 12.3 & 22.4 \\
1.0 & 12.7 & 32.1 \\
1.2 & 13.6 & 39.6 \\
\hline
\end{tabular}

Table S5: Model Parameters

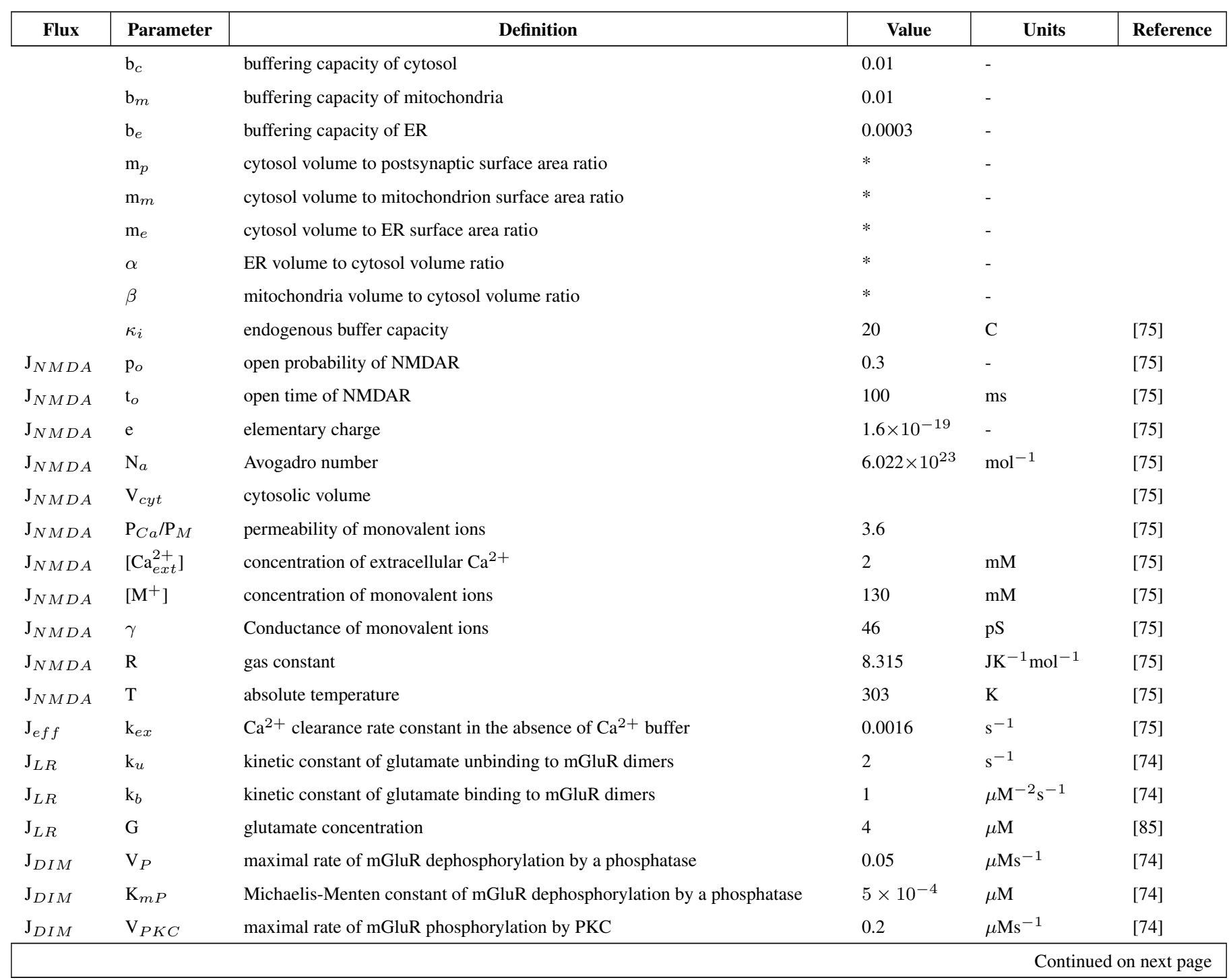


Table S5 - continued from previous page

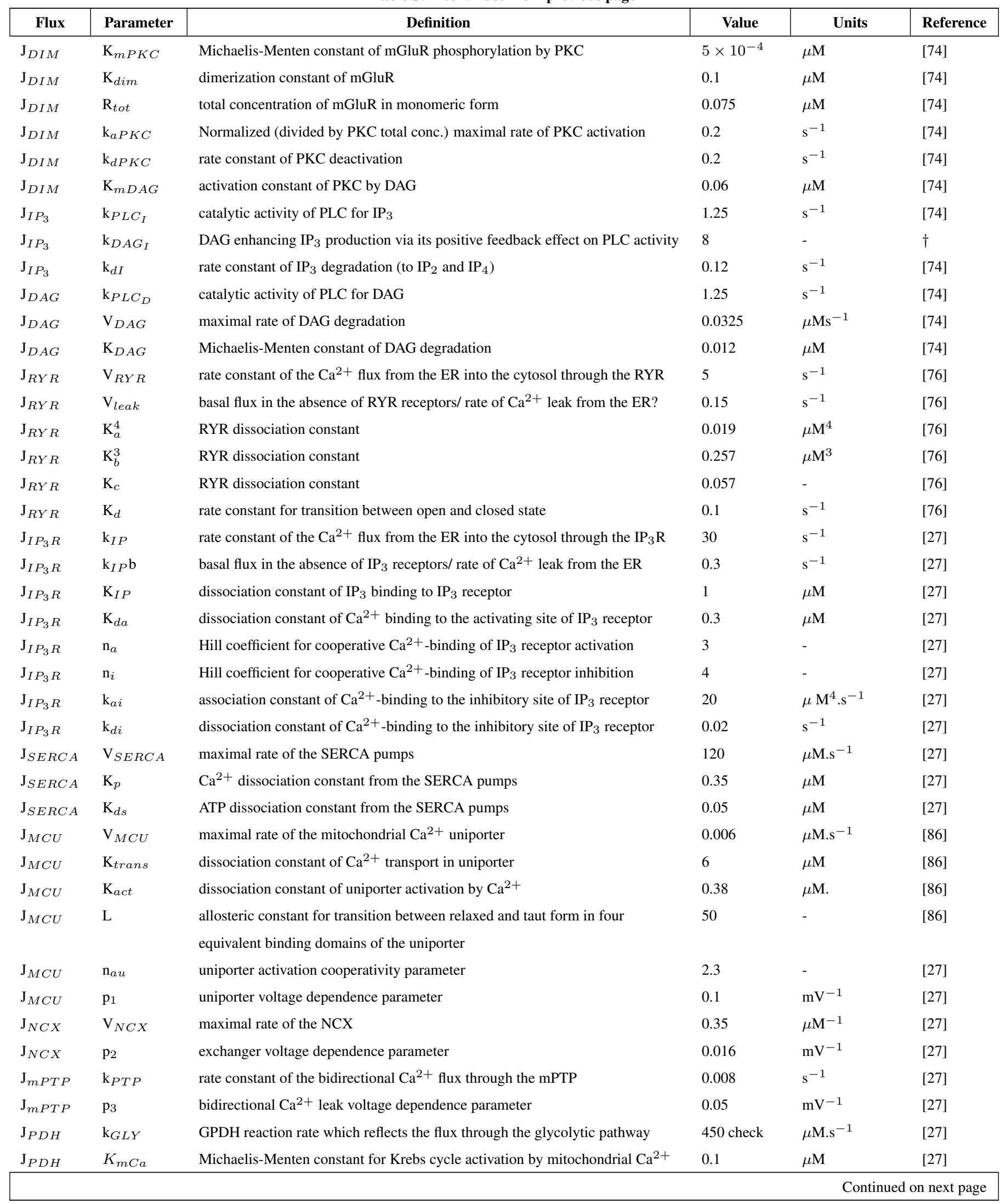


Table S5 - continued from previous page

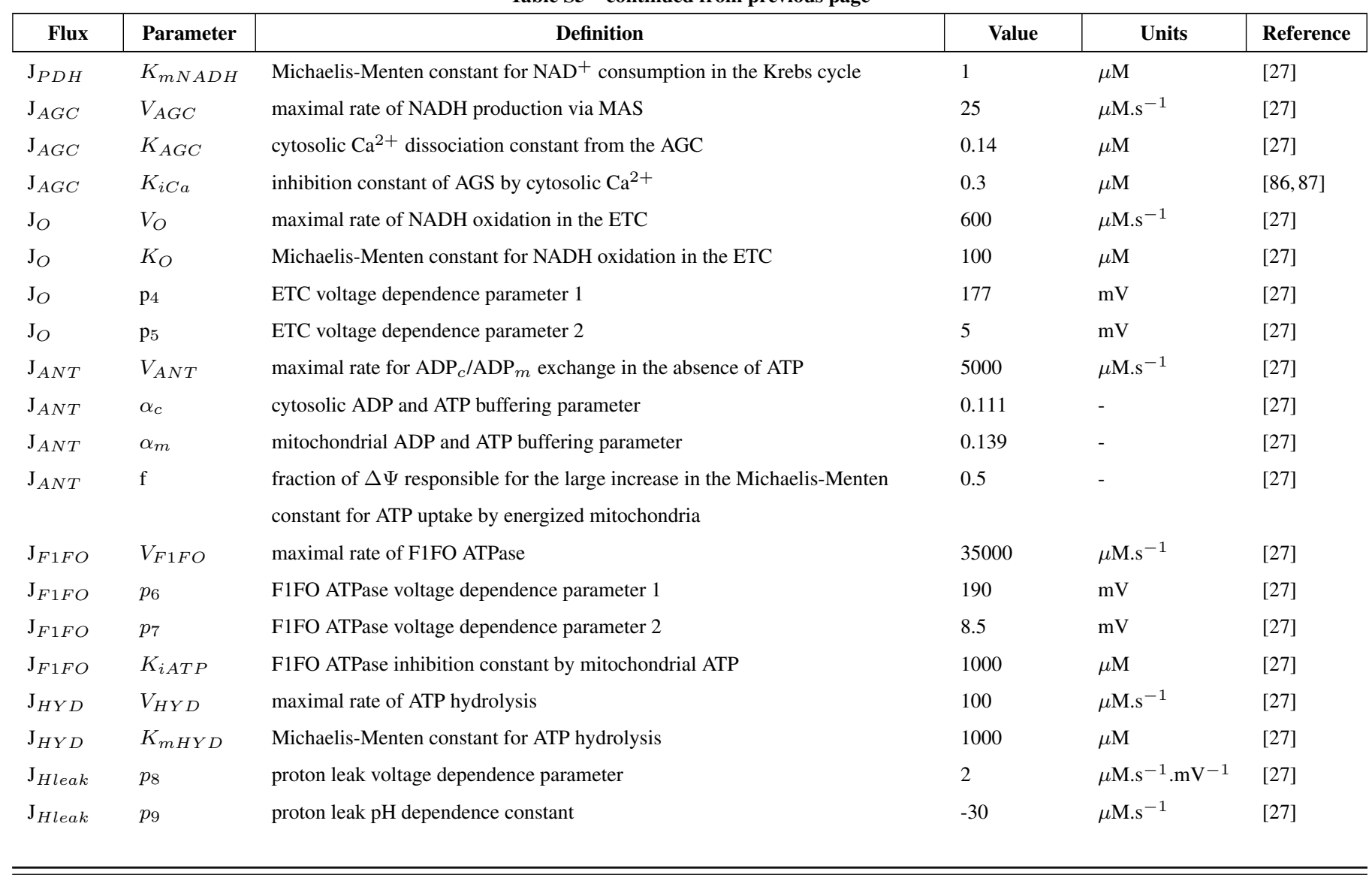


bioRxiv preprint doi: https://doi.org/10.1101/2020.09.12.294827; this version posted September 13, 2020. The copyright holder for this preprint (which was not certified by peer review) is the author/funder. All rights reserved. No reuse allowed without permission.

\section{Supplementary Figures}



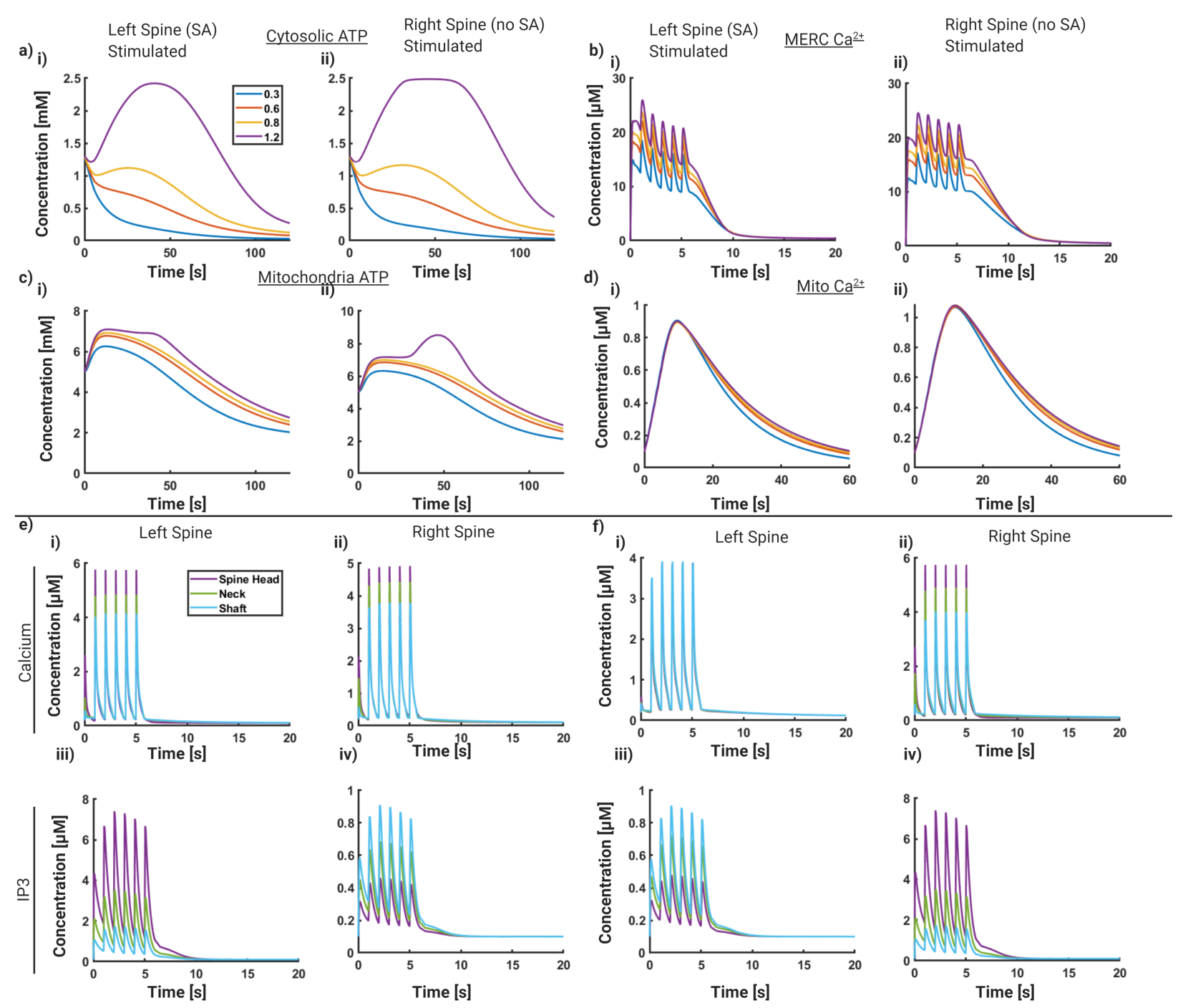

Left Spine (SA) Stimulated

Right Spine (no SA) Stimulated

Figure S1: Comparison between stimulus site for larger spine with apparatus and smaller spine with-

out apparatus a) For 4 different mitochondrial sizes, i) shows the volume averaged cytosolic ATP concentration when the spine with spine apparatus is stimulated (left spine) and ii) shows the average cytosolic ATP concentration when the spine without spine apparatus is stimulated (right spine) b) Same as a) for volume averaged MERC calcium concentration c) Same as a) for volume averaged mitochondrial ATP concentration d) Same as a) for volume averaged mitochondrial calcium e) For a single mitochondria size (0.6 micron), we show the stimulated spine and neighboring spine dynamics when the left spine (with SA) is stimulated for: i) calcium concentrations for 3 points in left spine head cytosol, ii) IP3 concentrations for 3 points in the left spine head cytosol, iii) calcium concentrations for 3 points in the right spine head cytosol, and iv) $\mathrm{IP}_{3}$ concentrations for 3 points in the right spine head cytosol. f) Same as e) for when the spine on the right with no spine apparatus is stimulated. 
a) NMDA only -contact

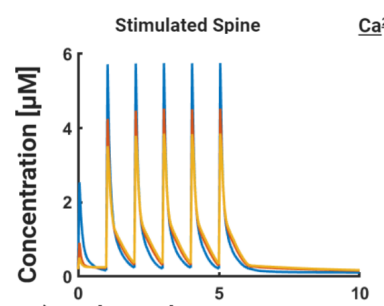

c) mGluR only -contact
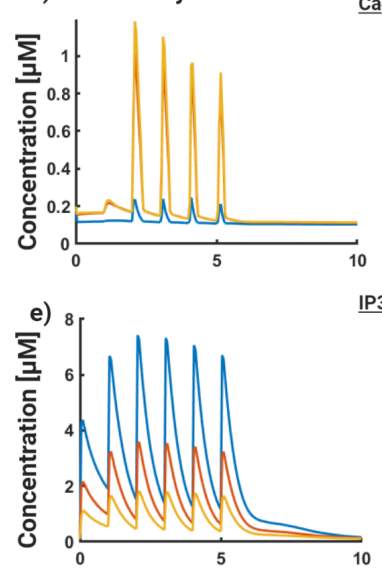

g) Full Model-contact
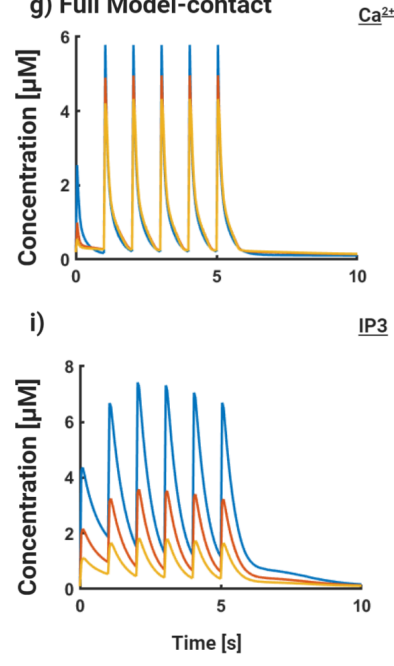
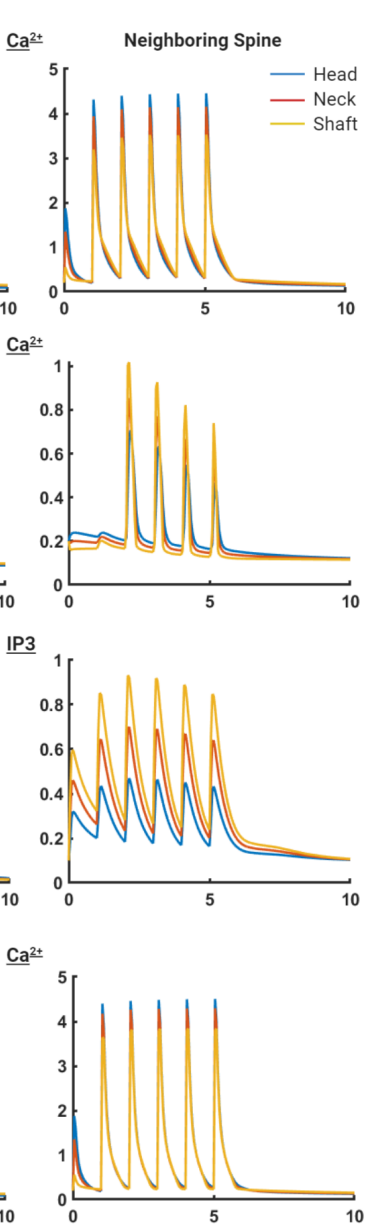

IP3

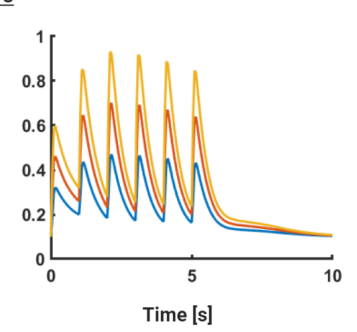

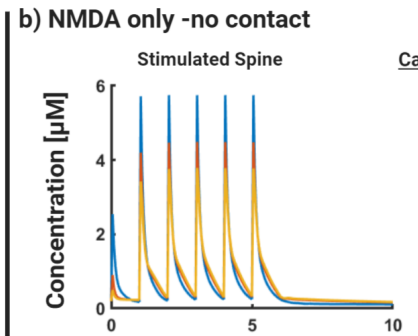
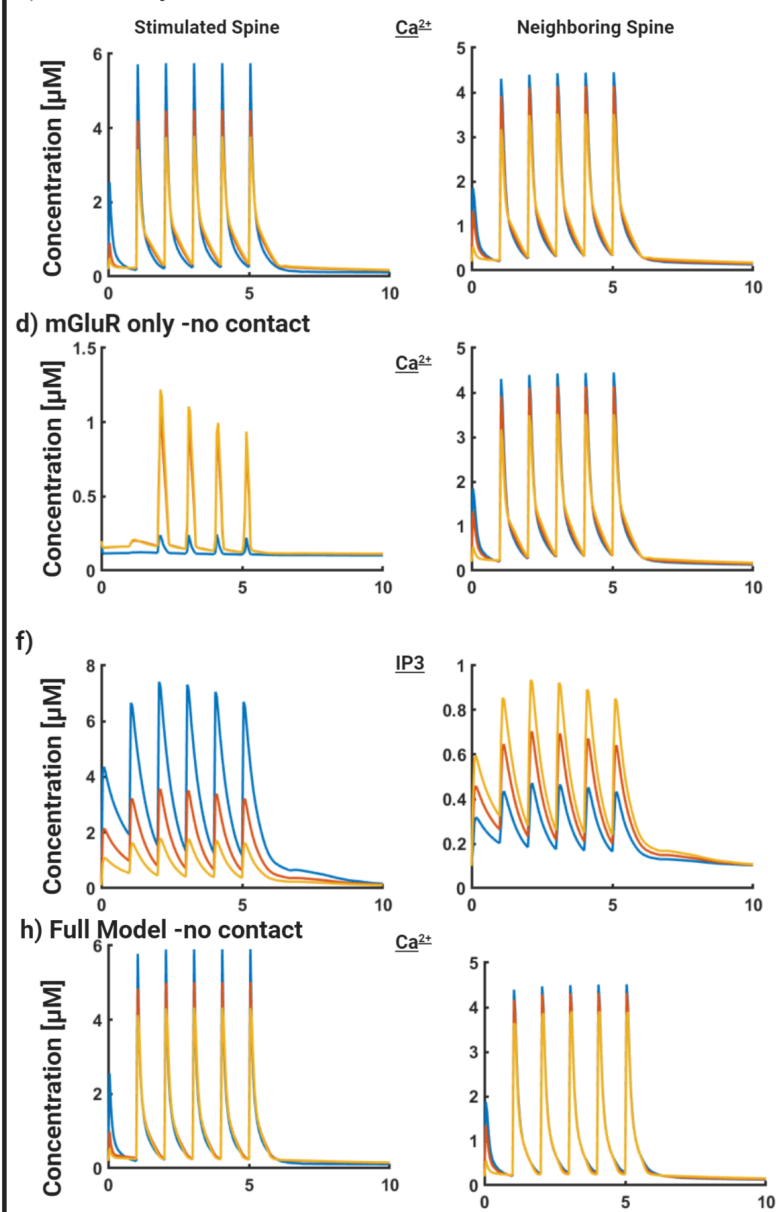

$\underline{\mathrm{Ca}^{2+}}$
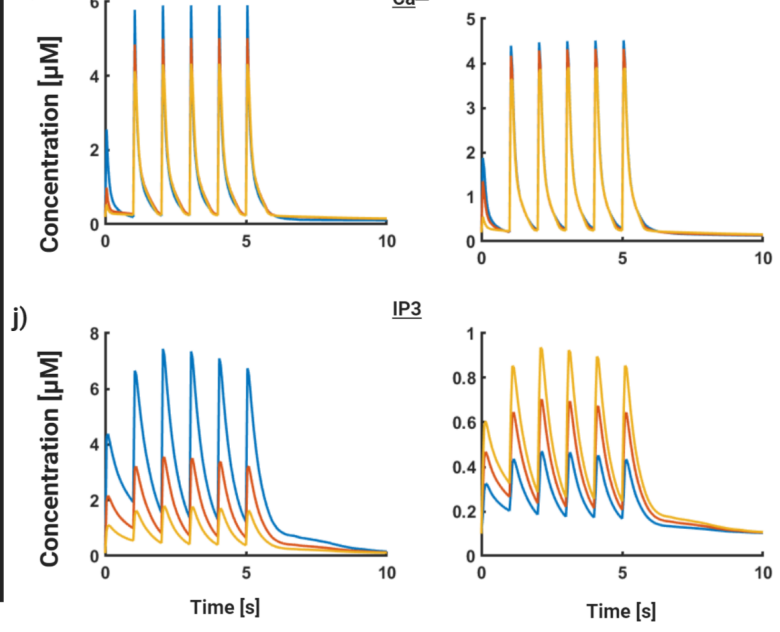

IP3

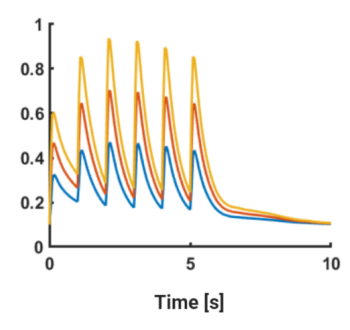

Figure S2: $\mathrm{Ca}^{2+}$ and $\mathrm{IP}_{3}$ Dynamics during stimulus from mGluR, NMDAR, and both models a) Calcium dynamics taken at 3 points in both the stimulated and neighboring spine in NMDAR-only system with MERC. b) same as a) for no MERC. c) Calcium dynamics taken at 3 points in both the stimulated and neighboring spine in mGluR-only system with MERC. d) Same as c) with no MERC. e)IP 3 dynamics taken at 3 points in both stimulated and neighboring spine in mGluR-only system with MERC. f) Same as e) with no MERC. g) Calcium dynamics taken at 3 points in both the stimulated and neighboring spine in combined system with MERC. h) Same as $\mathbf{g}$ ) with no MERC. i)IP ${ }_{3}$ dynamics taken at 3 points in both stimulated and neighboring spine in combined system with MERC. j) Same as i) with no MERC. 


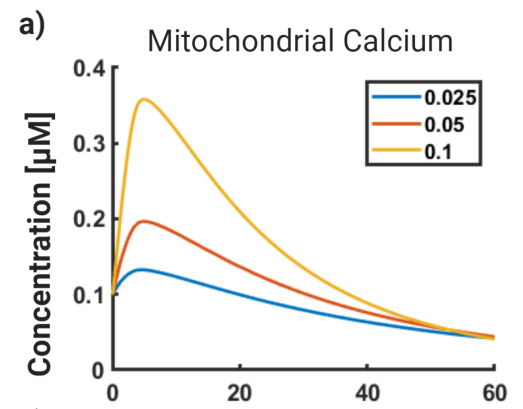

c)

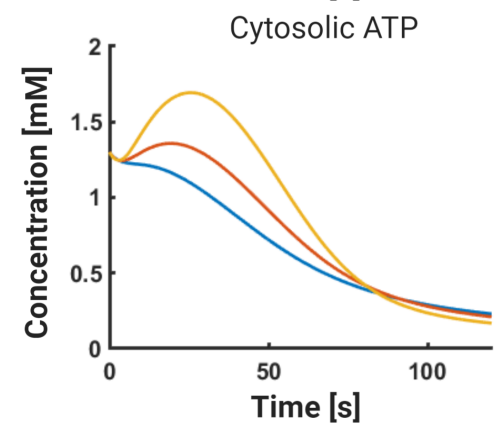

b)

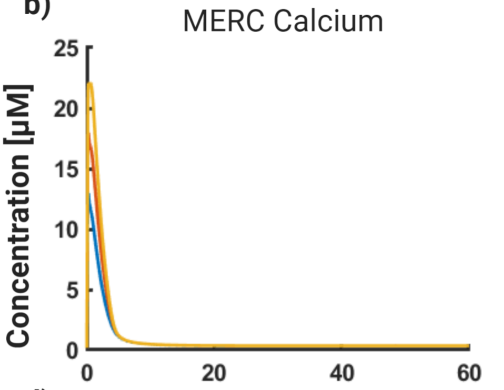

d)

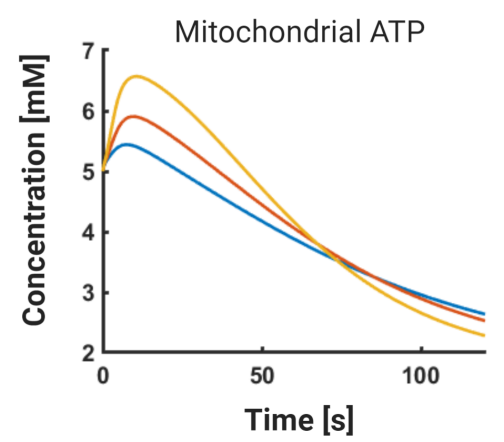

e) $1 \mathrm{~s}$

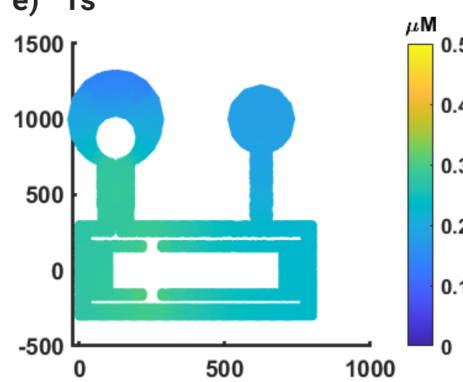

f) $5.1 \mathrm{~s}$

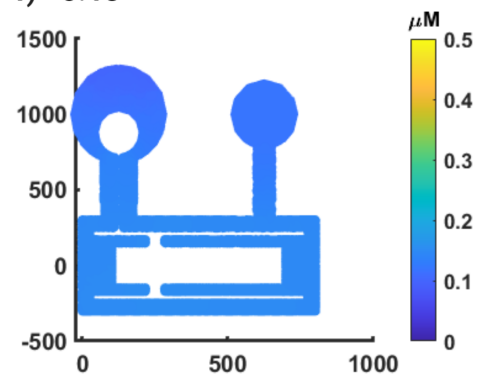

Figure S3: Simulations without glutamate response quickly decay toward steady-state a)Mitochondrial calcium dynamics with no stimulus for MERC covering 2.5, 5, and $10 \%$ mitochondria surface area. b) Same as a) for MERC Calcium. c) Same as a) for Cytosolic ATP. d) Same as a) for Mitochondrial ATP. e) 2D Spatial plot of cytosolic calcium with no stimulus at 1 second. f) same as e, but taken at 5.1s 

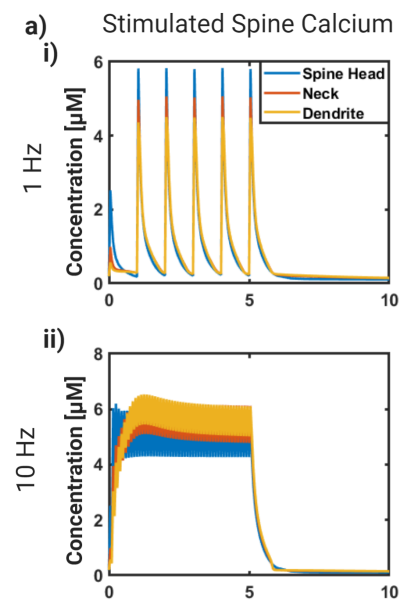

iii)

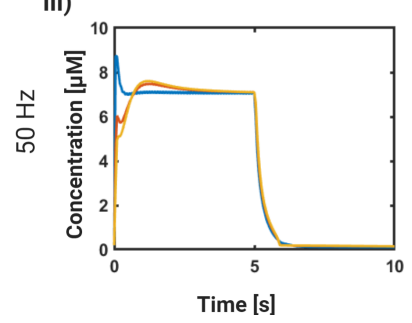

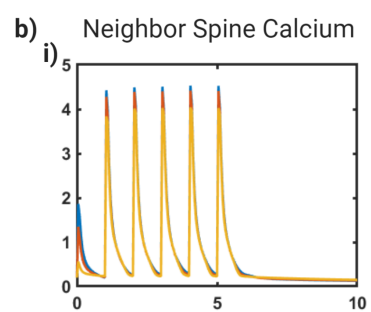

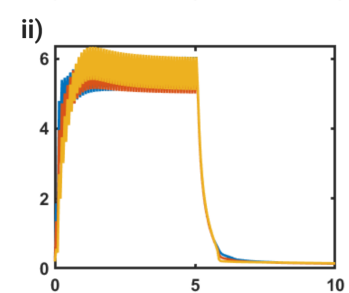

iii)

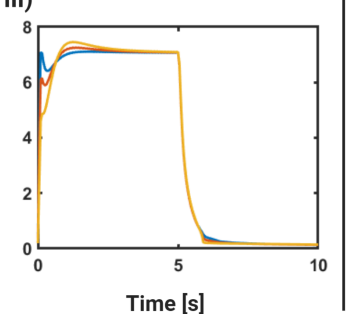

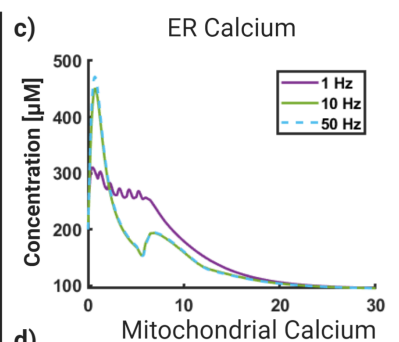

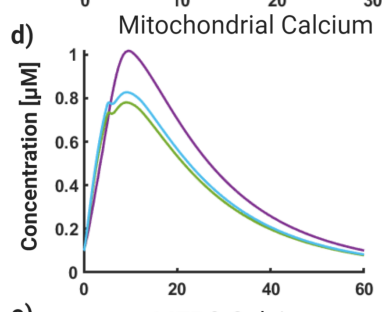

e)

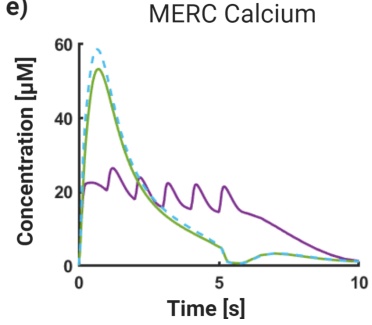

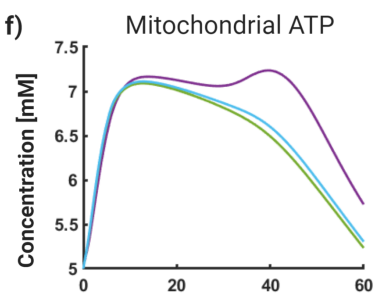
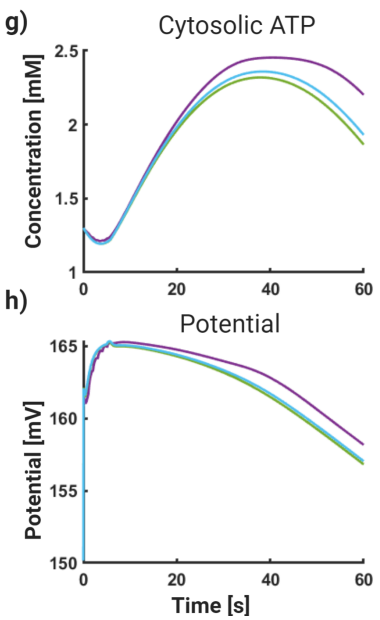

Figure S4: Changing stimulus frequency within range of expected signaling frequencies yields changes

in system behavior All results generated with mitochondria size of $0.6 \mu \mathrm{m}$ and 10 percent MERC surface area. a) Stimulated spine calcium concentrations at 3 points in the spine cytosol for the following frequencies: i) $1 \mathrm{~Hz}$ iib) $10 \mathrm{~Hz}$ iiib) $50 \mathrm{~Hz}$ b) Neighboring spine calcium concentrations at 3 points in the spine cytosols for ib) $1 \mathrm{~Hz}$, iib) $10 \mathrm{~Hz}$, iiib) $50 \mathrm{~Hz}$. c) Volume average ER Calcium for the 3 frequencies. $10 \mathrm{~Hz}$ and $50 \mathrm{~Hz}$ frequency plots overlay. d) Same as c) for Mitochondrial Calcium. e) Same as c) for MERC Calcium. f) Same as c) for Mitochondrial ATP. g) Same as c) for Cytosolic ATP. h) Same as c) for Mitochondrial Potential 
a)
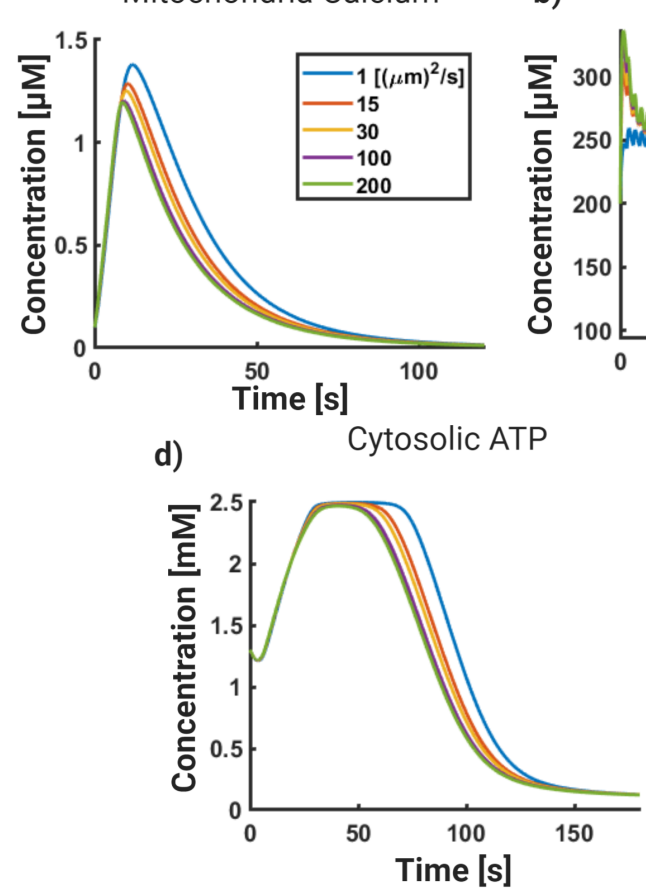

ER Calcium

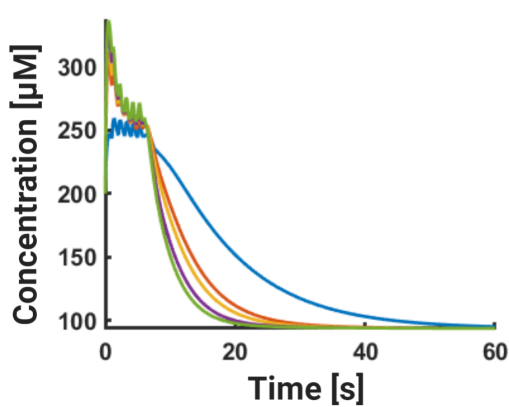

e) c) MERC Calcium

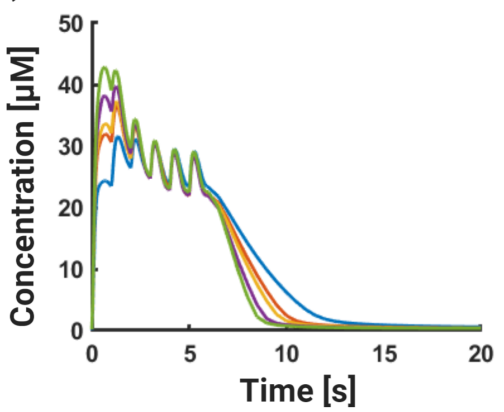

Mitochondrial ATP

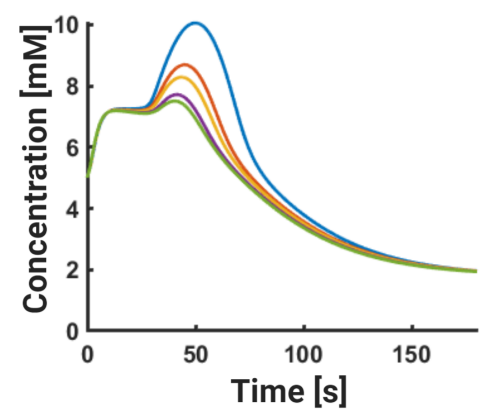

Figure S5: Calcium diffusion coefficient exhibits minor changes in system dynamics a) Mitochondrial dynamics in response to $51 \mathrm{~Hz}$ pulses varying diffusion constants of free calcium. b) ER calcium dynamics with varying calcium diffusion constants. c) MERC calcium dynamics with varying calcium diffusion constants. d) Cytosolic ATP dynamics with varying calcium diffusion constants. e) Mitochondria ATP dynamics with varying calcium diffusion constants. 

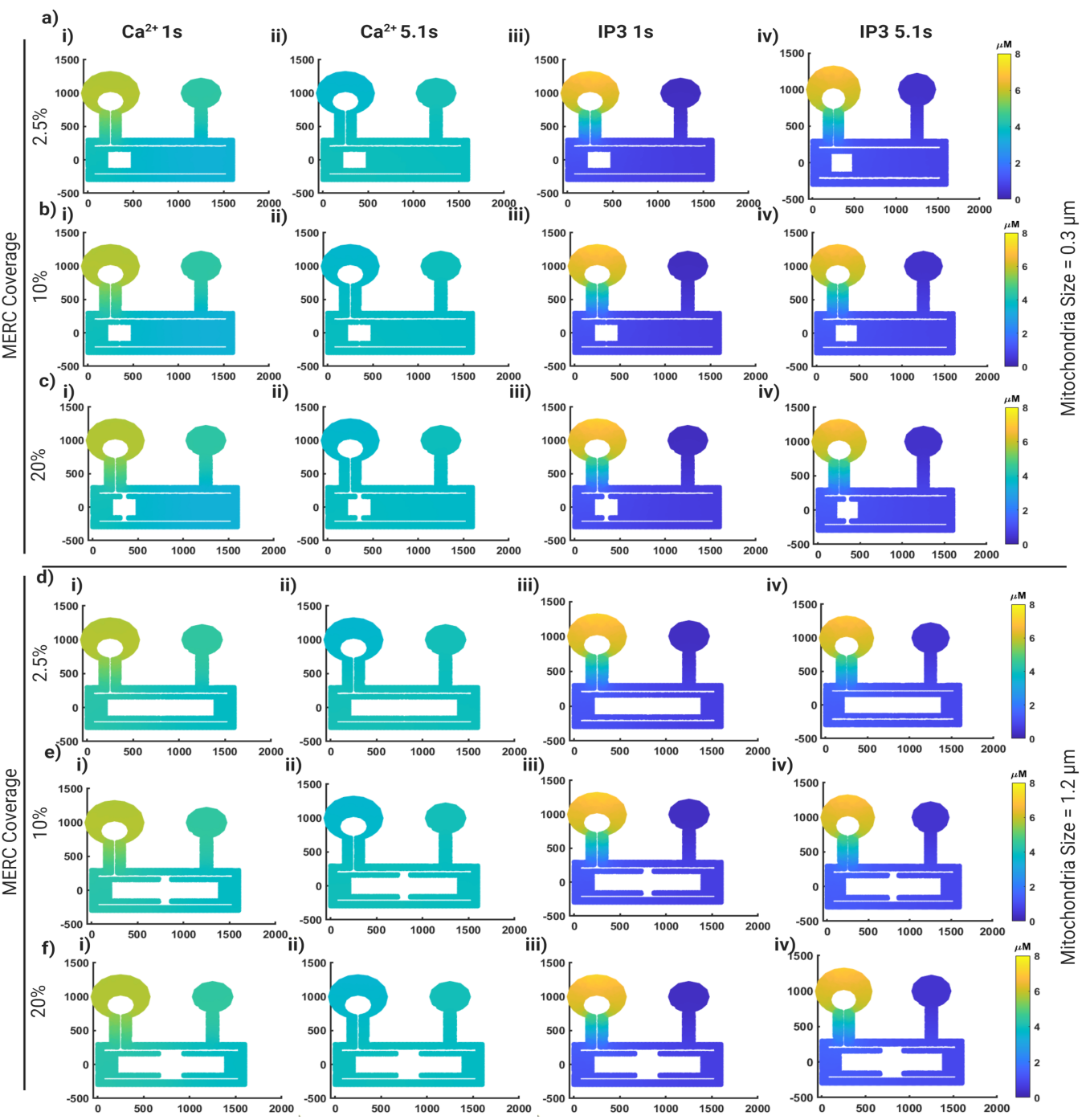

Figure S6: Cytosolic gradients do not change with changes in mitochondrial size or MERC surface

area coverage a) 2D Spatial plots for concentrations, in $\mu M$, for i) calcium at 1 second, ii) calcium at 5.1 seconds, iii) IP3 at 1 second, and iv) IP3 at 5.1 seconds for a mitochondrial size of $0.3 \mu \mathrm{m}$ and $2.5 \%$ of mitochondria surface area covered by MERC. b) Same as afor a mitochondria size of $0.3 \mu \mathrm{m}$ and $10 \%$ of mitochondria surface area covered by MERC. c) Same as afor a mitochondria size of $0.3 \mu \mathrm{m}$ and $20 \%$ of mitochondria surface area covered by MERC. d) Same as afor a mitochondria size of $1.2 \mu \mathrm{m}$ and $2.5 \%$ of mitochondria surface area covered by MERC. e) Same as afor a mitochondria size of $1.2 \mu \mathrm{m}$ and $10 \%$ of mitochondria surface area covered by MERC. f) Same as afor a mitochondria size of $1.2 \mu \mathrm{m}$ and $20 \%$ of mitochondria surface area covered by MERC. 
a)

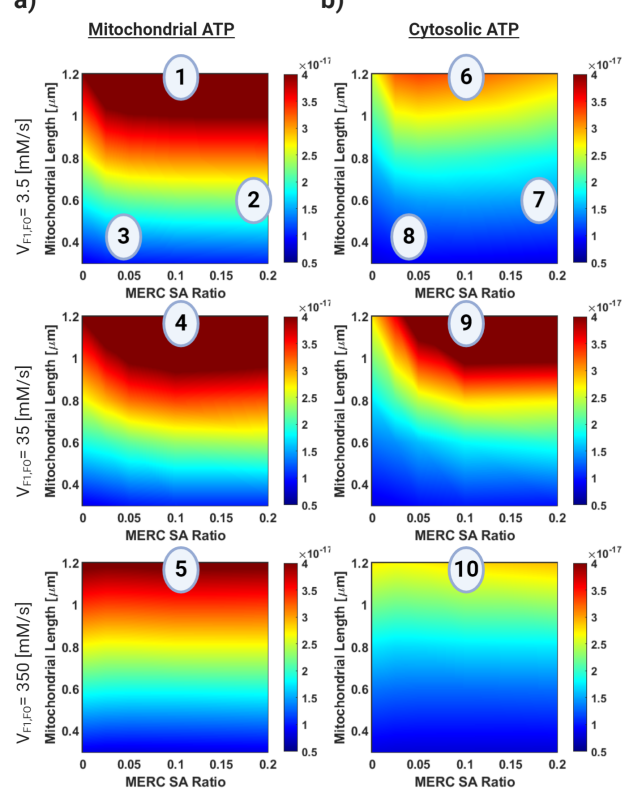

c)

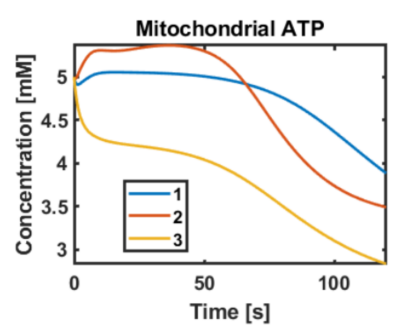

e)

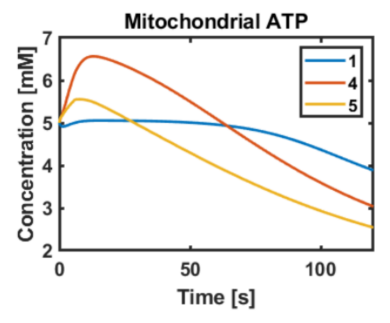

d)

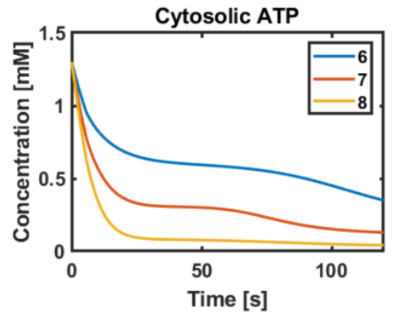

f)

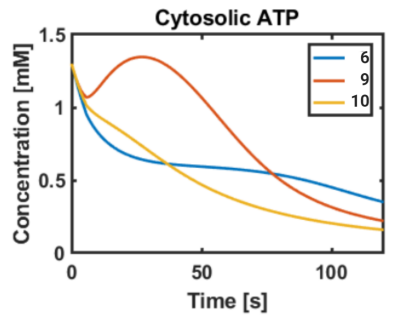

Figure S7: Dynamic plots for phase diagram show differential ATP generation a) Phase diagrams for the change in $\mathrm{V}_{F 1 F O}$ parameter (shown in Figure 6) for mitochondrial ATP. Here we denote 5 points on the phase diagram and explore the corresponding dynamics in $\mathbf{c}$ ) and d). b) Phase diagrams for the change in $\mathrm{V}_{F 1 F O}$ parameter (shown in Figure 6) for cytosolic ATP. Here we denote the same 5 points and explore the corresponding dynamics in e) and f). c) Comparison between 3 different phases displayed on the mitochondrial ATP phase diagram. d) Comparison between 3 different phases displayed on the cytosolic ATP phase diagram. The three dynamic plots behave similarly, but at different magnitudes. e) Comparison between the highest phases displayed on 3 different phase diagrams for mitochondrial ATP. Although all three diagrams have similar max AUC, higher $\mathrm{V}_{F 1 F O}$ values correspond to a higher initial production in this model. f) Comparison between the highest phases displayed on 3 different phase diagrams for mitochondrial ATP. 


\section{References}

[1] M. B. Robinson and J. T. Coyle, "Glutamate and related acidic excitatory neurotransmitters: from basic science to clinical application,” $F A S E B$ J., vol. 1, pp. 446-455, Dec. 1987.

[2] P. Jadiya, D. W. Kolmetzky, D. Tomar, A. Di Meco, A. A. Lombardi, J. P. Lambert, T. S. Luongo, M. H. Ludtmann, D. Praticò, and J. W. Elrod, "Impaired mitochondrial calcium efflux contributes to disease progression in models of Alzheimer's disease," Nature Communications, vol. 10, p. 3885, dec 2019 .

[3] R. C. Malenka, "The role of postsynaptic calcium in the induction of long-term potentiation," Mol. Neurobiol., vol. 5, no. 2-4, pp. 289-295, 1991.

[4] The MIT Press, "Dendritic spines." https://mitpress.mit.edu/books/dendritic-spines. Accessed: 2020-4-14.

[5] J. Larson and E. Munkácsy, “Theta-burst LTP,” Brain Res., vol. 1621, pp. 38-50, Sept. 2015.

[6] M. J. Higley and B. L. Sabatini, "Calcium Signaling in Dendritic Spines," Cold Spring Harbor Perspectives in Biology, vol. 4, pp. a005686a005686, apr 2012.

[7] M. Segal and E. Korkotian, "Endoplasmic reticulum calcium stores in dendritic spines," Frontiers in Neuroanatomy, vol. 8, p. 64, jul 2014.

[8] K. Basnayake, D. Mazaud, A. Bemelmans, N. Rouach, E. Korkotian, and D. Holcman, "Fast calcium transients in dendritic spines driven by extreme statistics," PLOS Biology, vol. 17, p. e2006202, jun 2019.

[9] P. Jedlicka, A. Vlachos, S. W. Schwarzacher, and T. Deller, "A role for the spine apparatus in LTP and spatial learning," Behav. Brain Res., vol. 192, pp. 12-19, Sept. 2008.

[10] V. Rangaraju, M. Lauterbach, and E. M. Schuman, "Spatially Stable Mitochondrial Compartments Fuel Local Translation during Plasticity," Cell, vol. 176, pp. 73-84.e15, jan 2019.

[11] Z.-X. Fu, X. Tan, H. Fang, P.-M. Lau, X. Wang, H. Cheng, and G.-Q. Bi, "Dendritic mitoflash as a putative signal for stabilizing long-term synaptic plasticity," Nature Communications, vol. 8, p. 31, dec 2017.

[12] M. E. Chicurel and K. M. Harris, "Three-dimensional analysis of the structure and composition of CA3 branched dendritic spines and their synaptic relationships with mossy fiber boutons in the rat hippocampus," J. Comp. Neurol., vol. 325, pp. 169-182, Nov. 1992.

[13] P. V. Martino Adami, Z. Nichtová, D. B. Weaver, A. Bartok, T. Wisniewski, D. R. Jones, S. Do Carmo, E. M. Castaño, A. C. Cuello, G. Hajnóczky, and L. Morelli, "Perturbed mitochondria-ER contacts in live neurons that model the amyloid pathology of alzheimer's disease," J. Cell Sci., vol. 132, Oct. 2019.

[14] S. Paillusson, R. Stoica, P. Gomez-Suaga, D. H. W. Lau, S. Mueller, T. Miller, and C. C. J. Miller, “There's something wrong with my MAM; the ER-Mitochondria axis and neurodegenerative diseases," Trends Neurosci., vol. 39, pp. 146-157, Mar. 2016.

[15] E. Area-Gomez, M. del Carmen Lara Castillo, M. D. Tambini, C. Guardia-Laguarta, A. J. C. de Groof, M. Madra, J. Ikenouchi, M. Umeda, T. D. Bird, S. L. Sturley, and E. A. Schon, "Upregulated function of mitochondria-associated ER membranes in alzheimer disease: Upregulated function of MAM in AD," EMBO J., vol. 31, pp. 4106-4123, Nov. 2012.

[16] L. Xu, X. Wang, and C. Tong, "Endoplasmic Reticulum-Mitochondria contact sites and neurodegeneration," Front Cell Dev Biol, vol. 8, p. 428 , June 2020 .

[17] G. Csordás, D. Weaver, and G. Hajnóczky, "Endoplasmic Reticulum-Mitochondrial Contactology: Structure and Signaling Functions,” Trends in Cell Biology, vol. 28, pp. 523-540, jul 2018.

[18] S. C. Alford, Y. Ding, T. Simmen, and R. E. Campbell, "Dimerization-dependent green and yellow fluorescent proteins," ACS Synth. Biol., vol. 1, pp. 569-575, Dec. 2012. 
[19] J. E. Vance, "MAM (mitochondria-associated membranes) in mammalian cells: lipids and beyond," Biochim. Biophys. Acta, vol. 1841, pp. 595-609, Apr. 2014.

[20] R. Bravo, J. M. Vicencio, V. Parra, R. Troncoso, J. P. Munoz, M. Bui, C. Quiroga, A. E. Rodriguez, H. E. Verdejo, J. Ferreira, M. Iglewski, M. Chiong, T. Simmen, A. Zorzano, J. A. Hill, B. A. Rothermel, G. Szabadkai, and S. Lavandero, "Increased ER-mitochondrial coupling promotes mitochondrial respiration and bioenergetics during early phases of ER stress,” J. Cell Sci., vol. 124, pp. 2511-2511, July 2011.

[21] S. Lee and K.-T. Min, “The interface between ER and mitochondria: Molecular compositions and functions," Mol. Cells, vol. 41, pp. 10001007, Dec. 2018.

[22] G. Mahajan and S. Nadkarni, "Intracellular calcium stores mediate metaplasticity at hippocampal dendritic spines," The Journal of Physiology, vol. 597, p. JP277726, jun 2019.

[23] L. F. Abbott and T. B. Kepler, "Model neurons: From Hodgkin-Huxley to hopfield," in Statistical Mechanics of Neural Networks, pp. 5-18, Springer Berlin Heidelberg, 1990.

[24] M. Bell, T. Bartol, T. Sejnowski, and P. Rangamani, "Dendritic spine geometry and spine apparatus organization govern the spatiotemporal dynamics of calcium,” J. Gen. Physiol., vol. 151, pp. 1017-1034, Aug. 2019.

[25] R. Bertram, M. Gram Pedersen, D. S. Luciani, and A. Sherman, "A simplified model for mitochondrial ATP production," Journal of Theoretical Biology, vol. 243, pp. 575-586, dec 2006.

[26] J. M. Han and V. Periwal, "A mathematical model of calcium dynamics: Obesity and mitochondria-associated ER membranes," 2018.

[27] B. Wacquier, L. Combettes, G. T. Van Nhieu, and G. Dupont, "Interplay Between Intracellular Ca2+ Oscillations and Ca2+-stimulated Mitochondrial Metabolism," Scientific Reports, vol. 6, p. 19316, may 2016.

[28] G. Magnus and J. Keizer, "Minimal model of beta-cell mitochondrial Ca2+ handling," American Journal of Physiology-Cell Physiology, vol. 273, pp. C717-C733, aug 1997.

[29] D. S. Luciani, S. Misler, and K. S. Polonsky, "Ca2+ controls slow NAD(P)H oscillations in glucose-stimulated mouse pancreatic islets," J. Physiol., vol. 572, pp. 379-392, Apr. 2006.

[30] H. Qi, L. Li, and J. Shuai, "Optimal microdomain crosstalk between endoplasmic reticulum and mitochondria for ca2+ oscillations," Sci. Rep., vol. 5, p. 7984, Jan. 2015.

[31] A. Cugno, T. M. Bartol, T. J. Sejnowski, R. Iyengar, and P. Rangamani, "Geometric principles of second messenger dynamics in dendritic spines," Sci. Rep., vol. 9, p. 11676, Aug. 2019.

[32] P. Rangamani, A. Lipshtat, E. U. Azeloglu, R. C. Calizo, M. Hu, S. Ghassemi, J. Hone, S. Scarlata, S. R. Neves, and R. Iyengar, "Decoding information in cell shape," Cell, vol. 154, pp. 1356-1369, Sept. 2013.

[33] R. C. Calizo, A. Ron, M. Hu, S. Bhattacharya, W. G. M. Janssen, J. Hone, S. Scarlata, P. Rangamani, and R. Iyengar, "Cell shape regulates subcellular organelle location to control short-term ca2+ signal dynamics in VSMC.” Oct. 2018.

[34] S. R. Neves, P. Tsokas, A. Sarkar, E. A. Grace, P. Rangamani, S. M. Taubenfeld, C. M. Alberini, J. C. Schaff, R. D. Blitzer, I. I. Moraru, and R. Iyengar, "Cell shape and negative links in regulatory motifs together control spatial information flow in signaling networks," Cell, vol. 133, pp. 666-680, May 2008.

[35] J. Meyers, J. Craig, and D. J. Odde, “Potential for control of signaling pathways via cell size and shape,” Curr. Biol., vol. 16, pp. 1685-1693, Sept. 2006.

[36] G. C. Brown and B. N. Kholodenko, "Spatial gradients of cellular phospho-proteins," FEBS Lett., vol. 457, pp. 452-454, Sept. 1999. 
[37] T. M. Bartol, D. X. Keller, J. P. Kinney, C. L. Bajaj, K. M. Harris, T. J. Sejnowski, and M. B. Kennedy, "Computational reconstitution of spine calcium transients from individual proteins," Front. Synaptic Neurosci., vol. 7, p. 17, Oct. 2015.

[38] K. T. Blackwell, "An efficient stochastic diffusion algorithm for modeling second messengers in dendrites and spines," J. Neurosci. Methods, vol. 157, pp. 142-153, Oct. 2006.

[39] R. Luján, Z. Nusser, J. D. B. Roberts, R. Shigemoto, and P. Somogyi, "Perisynaptic Location of Metabotropic Glutamate Receptors mGluR1 and mGluR5 on Dendrites and Dendritic Spines in the Rat Hippocampus," European Journal of Neuroscience, vol. 8, pp. 1488-1500, jul 1996.

[40] G. Santulli, R. Nakashima, Q. Yuan, and A. R. Marks, "Intracellular calcium release channels: an update,” J. Physiol., vol. 595, pp. 30413051, May 2017.

[41] C. Giorgi, S. Marchi, and P. Pinton, “The machineries, regulation and cellular functions of mitochondrial calcium," Nature Reviews Molecular Cell Biology, vol. 19, pp. 713-730, nov 2018.

[42] M. Dougoud, L. Vinckenbosch, C. Mazza, B. Schwaller, and L. Pecze, “The Effect of Gap Junctional Coupling on the Spatiotemporal Patterns of Ca2+ Signals and the Harmonization of Ca2+-Related Cellular Responses," PLOS Computational Biology, vol. 12, p. e1005295, dec 2016.

[43] C. Cserép, B. Pósfai, A. D. Schwarcz, and Á. Dénes, "Mitochondrial Ultrastructure Is Coupled to Synaptic Performance at Axonal Release Sites," eneuro, vol. 5, pp. ENEURO.0390-17.2018, jan 2018.

[44] Z.-H. Sheng and Q. Cai, "Mitochondrial transport in neurons: impact on synaptic homeostasis and neurodegeneration," Nature Reviews Neuroscience, vol. 13, pp. 77-93, feb 2012.

[45] R. Rizzuto, P. Pinton, W. Carrington, F. S. Fay, K. E. Fogarty, L. M. Lifshitz, R. A. Tuft, and T. Pozzan, "Close contacts with the endoplasmic reticulum as determinants of mitochondrial ca2+ responses," Science, vol. 280, pp. 1763-1766, June 1998.

[46] K.-S. Lee, S. Huh, S. Lee, Z. Wu, A.-K. Kim, H.-Y. Kang, and B. Lu, “Altered ER-mitochondria contact impacts mitochondria calcium homeostasis and contributes to neurodegeneration in vivo in disease models," Proceedings of the National Academy of Sciences, vol. 115, pp. E8844-E8853, sep 2018.

[47] S. S. Divakaruni, A. M. Van Dyke, R. Chandra, T. A. LeGates, M. Contreras, P. A. Dharmasri, H. N. Higgs, M. K. Lobo, S. M. Thompson, and T. A. Blanpied, "Long-Term potentiation requires a rapid burst of dendritic mitochondrial fission during induction," Neuron, vol. 100, pp. 860-875.e7, Nov. 2018.

[48] Y. Wu, C. Whiteus, C. S. Xu, K. J. Hayworth, R. J. Weinberg, H. F. Hess, and P. De Camilli, "Contacts between the endoplasmic reticulum and other membranes in neurons," Proceedings of the National Academy of Sciences, vol. 114, pp. E4859-E4867, jun 2017.

[49] A. Sood, D. V. Jeyaraju, J. Prudent, A. Caron, P. Lemieux, H. M. McBride, M. Laplante, K. Tóth, and L. Pellegrini, "A mitofusin-2-dependent inactivating cleavage of opa1 links changes in mitochondria cristae and ER contacts in the postprandial liver," Proc. Natl. Acad. Sci. U. S. A., vol. 111, pp. 16017-16022, Nov. 2014.

[50] Y. Hirabayashi, S.-K. Kwon, H. Paek, W. M. Pernice, M. A. Paul, J. Lee, P. Erfani, A. Raczkowski, D. S. Petrey, L. A. Pon, and F. Polleux, “ER-mitochondria tethering by PDZD8 regulates Ca 2+ dynamics in mammalian neurons," Science, vol. 358, pp. 623-630, nov 2017.

[51] A. Bartok, D. Weaver, T. Golenár, Z. Nichtova, M. Katona, S. Bánsághi, K. J. Alzayady, V. K. Thomas, H. Ando, K. Mikoshiba, S. K. Joseph, D. I. Yule, G. Csordás, and G. Hajnóczky, "IP3 receptor isoforms differently regulate ER-mitochondrial contacts and local calcium transfer," Nat. Commun., vol. 10, p. 3726, Aug. 2019.

[52] G. C. Garcia, T. M. Bartol, S. Phan, E. A. Bushong, G. Perkins, T. J. Sejnowski, M. H. Ellisman, and A. Skupin, "Mitochondrial morphology provides a mechanism for energy buffering at synapses,” Sci. Rep., vol. 9, p. 18306, Dec. 2019.

[53] E. Engl and D. Attwell, "Non-signalling energy use in the brain,” J. Physiol., vol. 593, pp. 3417-3429, Aug. 2015. 
[54] E. J. Griffiths and G. A. Rutter, "Mitochondrial calcium as a key regulator of mitochondrial ATP production in mammalian cells," Biochim. Biophys. Acta, vol. 1787, pp. 1324-1333, Nov. 2009.

[55] Z.-X. Fu, X. Tan, H. Fang, P.-M. Lau, X. Wang, H. Cheng, and G.-Q. Bi, "Dendritic mitoflash as a putative signal for stabilizing long-term synaptic plasticity,” Nat. Commun., vol. 8, p. 31, June 2017.

[56] Z. Li and M. Sheng, "Caspases in synaptic plasticity," Mol. Brain, vol. 5, p. 15, May 2012.

[57] R. Chakrabarti, W.-K. Ji, R. V. Stan, J. de Juan Sanz, T. A. Ryan, and H. N. Higgs, "INF2-mediated actin polymerization at the ER stimulates mitochondrial calcium uptake, inner membrane constriction, and division,” J. Cell Biol., vol. 217, pp. 251-268, Jan. 2018.

[58] C. T. Lee, J. G. Laughlin, J. B. Moody, R. E. Amaro, J. A. McCammon, M. Holst, and P. Rangamani, "An Open-Source mesh generation platform for biophysical modeling using realistic cellular geometries," Biophys. J., vol. 118, pp. 1003-1008, Mar. 2020.

[59] C. T. Lee, J. G. Laughlin, N. Angliviel de La Beaumelle, R. E. Amaro, J. A. McCammon, R. Ramamoorthi, M. Holst, and P. Rangamani, "3D mesh processing using GAMer 2 to enable reaction-diffusion simulations in realistic cellular geometries," PLoS Comput. Biol., vol. 16, p. e1007756, Apr. 2020.

[60] B. L. Sabatini, M. Maravall, and K. Svoboda, "Ca2+ signaling in dendritic spines," Current Opinion in Neurobiology, vol. 11, pp. 349-356, jun 2001.

[61] D. B. Dorman, J. Jedrzejewska-Szmek, and K. T. Blackwell, "Inhibition enhances spatially-specific calcium encoding of synaptic input patterns in a biologically constrained model," Elife, vol. 7, Oct. 2018.

[62] M. Ordyan, T. Bartol, M. Kennedy, P. Rangamani, and T. Sejnowski, "Interactions between calmodulin and neurogranin govern the dynamics of CaMKII as a leaky integrator,” PLoS Comput. Biol., vol. 16, p. e1008015, July 2020.

[63] J. Gonzalez, I. S. Morales, D. M. Villarreal, and B. E. Derrick, "Low-frequency stimulation induces long-term depression and slow onset long-term potentiation at perforant path-dentate gyrus synapses in vivo," J. Neurophysiol., vol. 111, pp. 1259-1273, Mar. 2014.

[64] J. D. Clements, R. A. Lester, G. Tong, C. E. Jahr, and G. L. Westbrook, "The time course of glutamate in the synaptic cleft," Science, vol. 258, pp. 1498-1501, Nov. 1992.

[65] K. Matsui, N. Hosoi, and M. Tachibana, "Active role of glutamate uptake in the synaptic transmission from retinal nonspiking neurons," $J$. Neurosci., vol. 19, pp. 6755-6766, Aug. 1999.

[66] C. Benninger, J. Kadis, and D. A. Prince, “Extracellular calcium and potassium changes in hippocampal slices,” Brain Res., vol. 187, pp. 165182, Apr. 1980.

[67] M. Cooling, P. Hunter, and E. J. Crampin, “Modeling Hypertrophic IP3 Transients in the Cardiac Myocyte," Biophysical Journal, vol. 93, pp. 3421-3433, nov 2007.

[68] Professor of Computation and Neural Systems Christof Koch, C. Koch, and I. Segev, Methods in Neuronal Modeling: From Ions to Networks. MIT Press, 1998.

[69] U. Manor, S. Bartholomew, G. Golani, E. Christenson, M. Kozlov, H. Higgs, J. Spudich, and J. Lippincott-Schwartz, "A mitochondriaanchored isoform of the actin-nucleating spire protein regulates mitochondrial division," Elife, vol. 4, Aug. 2015.

[70] COMSOL, "Comsol Multiphysics Reference Manual (Version 5.4)," 2018.

[71] N. Holbro, A. Grunditz, and T. G. Oertner, "Differential distribution of endoplasmic reticulum controls metabotropic signaling and plasticity at hippocampal synapses," Proceedings of the National Academy of Sciences, vol. 106, pp. 15055-15060, sep 2009.

[72] J. I. Arellano, R. Benavides-Piccione, J. DeFelipe, and R. Yuste, "Ultrastructure of dendritic spines: correlation between synaptic and spine morphologies," Frontiers in Neuroscience, vol. 1, pp. 131-143, nov 2007. 
[73] A. A. Lombardi and J. W. Elrod, “Mediating ER-mitochondrial cross-talk,” Science, vol. 358, pp. 591-592, nov 2017.

[74] G. Dupont, E. F. Loomekandja Lokenye, and R. J. Challiss, "A model for Ca2+ oscillations stimulated by the type 5 metabotropic glutamate receptor: An unusual mechanism based on repetitive, reversible phosphorylation of the receptor," Biochimie, vol. 93, pp. 2132-2138, dec 2011.

[75] E. A. Nimchinsky, “The Number of Glutamate Receptors Opened by Synaptic Stimulation in Single Hippocampal Spines," Journal of Neuroscience, vol. 24, pp. 2054-2064, feb 2004.

[76] J. Keizer and L. Levine, "Ryanodine receptor adaptation and Ca2+(-)induced Ca2+ release-dependent Ca2+ oscillations," Biophysical Journal, vol. 71, pp. 3477-3487, dec 1996.

[77] R. Yasuda, "Biophysics of Biochemical Signaling in Dendritic Spines: Implications in Synaptic Plasticity," Biophysical Journal, vol. 113, pp. 2152-2159, nov 2017.

[78] G. Szabadkai and M. R. Duchen, "Mitochondria: The Hub of Cellular Ca 2+ Signaling," Physiology, vol. 23, pp. 84-94, apr 2008.

[79] R. Greget, F. Pernot, J.-M. C. Bouteiller, V. Ghaderi, S. Allam, A. F. Keller, N. Ambert, A. Legendre, M. Sarmis, O. Haeberle, M. Faupel, S. Bischoff, T. W. Berger, and M. Baudry, "Simulation of Postsynaptic Glutamate Receptors Reveals Critical Features of Glutamatergic Transmission," PLoS ONE, vol. 6, p. e28380, dec 2011.

[80] T. K. Rostovtseva and S. M. Bezrukov, "ATP Transport Through a Single Mitochondrial Channel, VDAC, Studied by Current Fluctuation Analysis,” Biophysical Journal, vol. 74, pp. 2365-2373, may 1998.

[81] E. Decrock, M. De Bock, N. Wang, A. K. Gadicherla, M. Bol, T. Delvaeye, P. Vandenabeele, M. Vinken, G. Bultynck, D. V. Krysko, and L. Leybaert, "IP3, a small molecule with a powerful message," Biochimica et Biophysica Acta (BBA) - Molecular Cell Research, vol. 1833, pp. 1772-1786, jul 2013.

[82] T. Manninen, R. Havela, and M.-L. Linne, "Reproducibility and Comparability of Computational Models for Astrocyte Calcium Excitability," Frontiers in Neuroinformatics, vol. 11, p. 11, feb 2017.

[83] S.-A. Brown, F. Morgan, J. Watras, and L. M. Loew, "Analysis of Phosphatidylinositol-4,5-Bisphosphate Signaling in Cerebellar Purkinje Spines," Biophysical Journal, vol. 95, pp. 1795-1812, aug 2008.

[84] D. G. Nicholls, S. Vesce, L. Kirk, and S. Chalmers, "Interactions between mitochondrial bioenergetics and cytoplasmic calcium in cultured cerebellar granule cells," Cell Calcium, vol. 34, pp. 407-424, oct 2003.

[85] Y. Okubo, H. Sekiya, S. Namiki, H. Sakamoto, S. Iinuma, M. Yamasaki, M. Watanabe, K. Hirose, and M. Iino, "Imaging extrasynaptic glutamate dynamics in the brain," Proceedings of the National Academy of Sciences, vol. 107, pp. 6526-6531, apr 2010.

[86] L. Contreras, P. Gomez-Puertas, M. Iijima, K. Kobayashi, T. Saheki, and J. Satrústegui, "Ca 2+ Activation Kinetics of the Two AspartateGlutamate Mitochondrial Carriers, Aralar and Citrin,” Journal of Biological Chemistry, vol. 282, pp. 7098-7106, mar 2007.

[87] C. B. Rueda, I. Llorente-Folch, I. Amigo, L. Contreras, P. González-Sánchez, P. Martínez-Valero, I. Juaristi, B. Pardo, A. del Arco, and J. Satrústegui, "Ca2+ regulation of mitochondrial function in neurons," Biochimica et Biophysica Acta (BBA) - Bioenergetics, vol. 1837, pp. 1617-1624, oct 2014. 